\title{
Stabilization of foams by the combined effects of an insoluble gas species and gelation
}

\author{
Houda Bey, ${ }^{\star a b}$ Frédéric Wintzenrieth, ${ }^{a}$ Olivier Ronsin, ${ }^{a}$ Reinhard $\mathrm{H}_{0} \mathrm{hler}^{\mathrm{ac}}$ and \\ Sylvie Cohen-Addad*ac \\ Liquid foams are unstable due to aging processes such as drainage, coalescence or coarsening. Since \\ these processes modify the foam structure, they can be a severe limitation to the elaboration of solid \\ foams with controlled structures inherited from their liquid precursors. Such applications call for a \\ thorough understanding of foam stabilization. Here we study how coarsening can be inhibited by the \\ combined effects of a mixture of gas containing a species insoluble in the foaming solution and of \\ gelation of the foaming solution. We present experiments with model ordered liquid foams and hydrogel \\ foams. They allow us to identify the underlying physical mechanisms of stabilization and their governing \\ parameters, namely the bubble radius $R_{0}$, the foam shear modulus $G$ and the number $\eta_{\circ}$ of insoluble \\ trapped gas molecules per bubble. We propose a scaling model that predicts the stability diagram of an \\ ideal monodisperse perfectly ordered foam as a function of $R_{0}, G$ and $\eta_{0}$, in qualitative agreement with \\ our data. We show that the domain of stable foams is governed by a characteristic elasto-capillary \\ radius set by the ratio of surface tension to storage modulus.
}

\section{Introduction}

Solid foams are cellular materials constituted of dense packings of gas cells in a solid matrix. They are made of a wide variety of materials including metals, glass, (bio-) ceramics or polymers. ${ }^{1-3}$ Their low density combined with their cellular structure considerably extends the range of physical, mechanical and thermal properties achievable for solid materials compared to that of dense solids and makes them sought after in many applications. ${ }^{1}$ For instance, they are used as packaging or construction building materials due to their thermal, acoustic and electrical insulating properties as well as their light weight. Solid foams are also used in chemical engineering applications as filters, carriers for chemical species or microporous membranes such as artificial skin. ${ }^{1}$ Foams made with hydrogels are beneficial to applications that require biocompatibility to elaborate absorbable sponges for wound dressing ${ }^{4}$ or scaffolds for tissue cell engineering ${ }^{5-7}$ for instance, and they have potential applications in green chemistry or green engineering such as surface decontamination processes. ${ }^{8}$

\footnotetext{
${ }^{a}$ Sorbonne Universités, UPMC Univ Paris 06, CNRS-UMR 7588, Institut des NanoSciences de paris, 4 place Jussieu, 75005 Paris, France. E-mail: sylvie.cohen-addad@insp.upmc.fr

${ }^{b}$ Université de Tunis El Manar, Faculté des Sciences de Tunis, LR99ES16 Laboratoire Physique de la Matière Molle et de la Modélisation électromagnétique, 2092, Tunis, Tunisia.E-mail: houda.bey@fst.utm.tn

${ }^{c}$ Université Paris-Est Marne-la-Vallée, 5 Bd Descartes, Champs-sur-Marne, 77454 Marne-la-Vallée cedex 2, France
}

Solid foams are elaborated from precursor liquid foams from whom they inherit their morphology. The final solid foam structure results from aging processes ${ }^{9}$ such as coalescence, drainage and coarsening that alter the liquid foam in the course of solidification. Therefore, stable liquid precursors, where aging is slowed down or even better arrested, ${ }^{10-12}$ are crucial to design solid foams with controlled bubble sizes, gas volume fraction and packing structure. Drainage can be arrested when using for instance a concentrated particle suspension as the continuous phase where the particles are jammed in the liquid interstices. ${ }^{13,14}$ Moreover, drainage and coalescence can be slowed down by using a foaming liquid ${ }^{8,11,15}$ with either large bulk viscosity or specific interfacial rheological properties $^{9,16}$ that provide large surface viscosity or surface elastic modulus. Stable highly ordered monodisperse solid foams have been obtained combining a milli-fluidic generator and a gelifying liquid phase. ${ }^{17,18}$ The conditions under which the dissolution of an isolated bubble in an elastic infinite matrix can be stopped have been analyzed theoretically. ${ }^{19}$ If the shear modulus of the matrix is large enough, its deformation that goes along with the bubble deflation or inflation generates elastic stresses large enough to counteract the effect of the capillary pressure. However, the elastic properties of the continuous phase minimally required for foam stabilization have so far not been clarified, and no physical model of this process is available.

Coarsening kinetics can be slowed down using different methods:

- a geometrical one, which consists of using monodisperse bubbles arranged in highly crystalline structures where the 
Laplace pressures in neighbouring bubbles are to a good approximation the same. ${ }^{20}$ However, such structures generally present defects which act as nucleation sites for coarsening. ${ }^{21}$

- a physicochemical one, where a foaming solution containing fatty acid co-surfactants is used. The dense surfactant monolayer adsorbed at the liquid-gas interface decreases the gas permeability of the liquid film. ${ }^{22}$ Alternatively, nanoparticles adsorbed at the interfaces can arrest coarsening as they form an elastic skin able to oppose mechanical resistance to the deformation of the interfaces. ${ }^{11,23}$

- another physicochemical method makes use of a gas mixture containing a small amount of a species which is insoluble in water. If the soluble gas diffuses out of a bubble that thus becomes smaller than its neighbours, the concentration of the insoluble species in this bubble increases, leading to the build up of an osmotic pressure that is opposed to the Laplace pressure. 2D numerical simulations on a dry disordered polydisperse foam structure (soap froths) have shown that coarsening can indeed be arrested by an insoluble species in the gas mixture, leading to a very heterogeneous stable structure. ${ }^{24}$ This effect is analogous to the inhibition of Ostwald ripening in a dilute emulsion by trapping in the droplets a molecular species insoluble in the continuous phase. ${ }^{25,26}$ Several experiments with liquid foams have shown empirically the efficiency of such gas mixtures to slow down coarsening, ${ }^{9,27,28}$ but the criterion for foam stabilization has not been established yet.

In this paper we study experimentally how coarsening of an initially monodisperse liquid foam can be inhibited by the combined effects of an insoluble gas species and gelation of the continuous phase, as a function of three control parameters: the bubble size $d$, the partial pressure of the insoluble species $P_{\eta}$ and the elastic modulus of the solid foam $G$. We identify a mechanism of stabilization based on the competition between the capillary pressure, the elastic stresses in the gel and the osmotic pressure due to the insoluble species. As a reference case, we first study the stabilisation solely due to the insoluble gas species. Then we show that, in gelled foams, in qualitative agreement with our data, the onset of coarsening is governed by a critical elasto-capillary bubble radius, set by the ratio of surface tension and foam shear modulus. We determine a semi-empirical criterion predicting for what bubble sizes, insoluble gas partial pressure and solid foam elasticity a foam is stable.

\section{Experimental section}

\subsection{Materials}

As a foaming agent, we use the nonionic surfactant Triton X100 (Sigma-Aldrich). Gelatin from porcine skin (type A) is purchased from Sigma-Aldrich. It has an average molar mass of $90 \mathrm{kDa}$ and its isoelectric point is in the range of $\mathrm{pH} \approx 7-9$. Sodium azide from Sigma-Aldrich is used as an antibacterial agent in the gelatin solution. Perfluorohexane is obtained from ABCR. All chemicals are used as received. Aqueous solutions are prepared using Ultrapure water (Millipore). Concentrations are defined as weight fractions.

\subsection{Foaming solutions}

Liquid foam samples are made from a foaming solution containing $0.45 \mathrm{wt} \%$ ( $\approx 35 \mathrm{cmc}$ ) of Triton X100 dissolved in water.

Gelatin foams are prepared from aqueous foaming solutions containing gelatin, sodium azide and Triton X100 as a surfactant. We vary the concentrations of Triton X100 and gelatin, denoted by $C$ and $C_{\text {gel }}$, respectively, keeping constant their ratios. First, a stock solution containing $9 \mathrm{wt} \%$ of Triton X100 in water is prepared, as well as a stock solution of sodium azide at $3 \mathrm{wt} \%$. Then gelatin powder, stock Triton solution and stock sodium azide solution are added to water heated at $60{ }^{\circ} \mathrm{C}$ in quantities necessary to achieve the final concentrations: $C=0.45 \mathrm{wt} \%$ with $C_{\text {gel }}=5 \mathrm{wt} \%$, or $C=0.90 \mathrm{wt} \%$ with $C_{\text {gel }}=10 \mathrm{wt} \%$. The sodium azide concentration is fixed at $0.036 \mathrm{wt} \%$. The solution is homogenized under gentle stirring at $T=60{ }^{\circ} \mathrm{C}$ for 2 hours. It is stored at $4{ }^{\circ} \mathrm{C}$ and heated at $50{ }^{\circ} \mathrm{C}$ just before use. The $\mathrm{pH}$ of these gelatin solutions is 5.3 .

We measure the surface tension $\gamma$ of the foaming solutions using a Wilhelmy plate tensiometer. In the absence of gelatin, for both Triton X100 concentrations, we find $\gamma=31.3 \mathrm{mN} \mathrm{m}^{-1}$ at $T=21 \pm 1{ }^{\circ} \mathrm{C}$, a value consistent with previous results, ${ }^{29}$ and $\gamma=30.2 \mathrm{mN} \mathrm{m}^{-1}$ at $T=45^{\circ} \mathrm{C}$. For both gelatin foaming solutions, we measure $\gamma=30.2 \mathrm{mN} \mathrm{m}^{-1}$ at $T=45{ }^{\circ} \mathrm{C}$. This indicates that gelatin has no impact on the surface tension of the liquid-gas interface in the studied ranges of Triton X100 and gelatin concentrations, consistently with previous work. ${ }^{29}$ We assume that our gelatin gels at $T=21{ }^{\circ} \mathrm{C}$ have the same interfacial tension as that of a liquid solution with the same Triton X100 concentration at the same temperature, namely $\gamma=31.3 \mathrm{mN} \mathrm{m}^{-1}$ at $T=21 \pm 1{ }^{\circ} \mathrm{C}$.

\subsection{Gas mixtures}

To slow down foam coarsening, which rapidly develops in foams containing nitrogen only, we use a mixture of nitrogen gas and perfluorohexane $\left(\mathrm{C}_{6} \mathrm{~F}_{14}\right)$ vapor that has an extremely low solubility in water. The solubility is characterized by the Henry's law solubility constant, $H=c / P$, where $c$ is the concentration of the dissolved gas in a bulk liquid at partial pressure $P$. We use the indices $\alpha$ and $\eta$ to distinguish the soluble (nitrogen) and the quasi-insoluble $\left(\mathrm{C}_{6} \mathrm{~F}_{14}\right)$ gas species. At $25{ }^{\circ} \mathrm{C}$ in water, we have ${ }^{30} H_{\alpha} \approx 6.4 \times 10^{-6} \mathrm{~mol} \mathrm{~m}{ }^{-3} \mathrm{~Pa}^{-1}$. This is four orders of magnitude larger than $H_{\eta} \approx 5.4 \times 10^{-10} \mathrm{~mol} \mathrm{~m}^{-3} \mathrm{~Pa}^{-1}$. The gas mixture is prepared with a controlled partial pressure of $\mathrm{C}_{6} \mathrm{~F}_{14}$, denoted as $P_{\eta}$, using the setup ${ }^{31,32}$ schematically shown in the red dashed frame in Fig. 1. A mixing chamber of volume $V_{\mathrm{c}}=1 \mathrm{~L}$ is evacuated to a pressure of $\approx 0.5$ mbar. A given volume $v$ of liquid $\mathrm{C}_{6} \mathrm{~F}_{14}$ is then injected through a septum into the chamber. Finally, nitrogen gas is added until the total pressure reaches $P_{\mathrm{c}}=2$ bar. The gas mixture is homogenized for several minutes using a fan placed inside the chamber. The molar volume of liquid $\mathrm{C}_{6} \mathrm{~F}_{14}$ at room temperature $T$ is $v_{\mathrm{m}}=$ $2.02 \times 10^{-4} \mathrm{~m}^{3}$. Upon foam generation, the gas expands isothermally and its pressure drops to the atmospheric pressure $P_{\mathrm{o}}$. The final composition of the gas mixture is characterized by its $\mathrm{C}_{6} \mathrm{~F}_{14}$ partial pressure $P_{\eta}=\left(P_{\mathrm{o}} / P_{\mathrm{c}}\right)\left(v / v_{\mathrm{m}}\right)\left(R T / V_{\mathrm{c}}\right)$, where $R$ is 


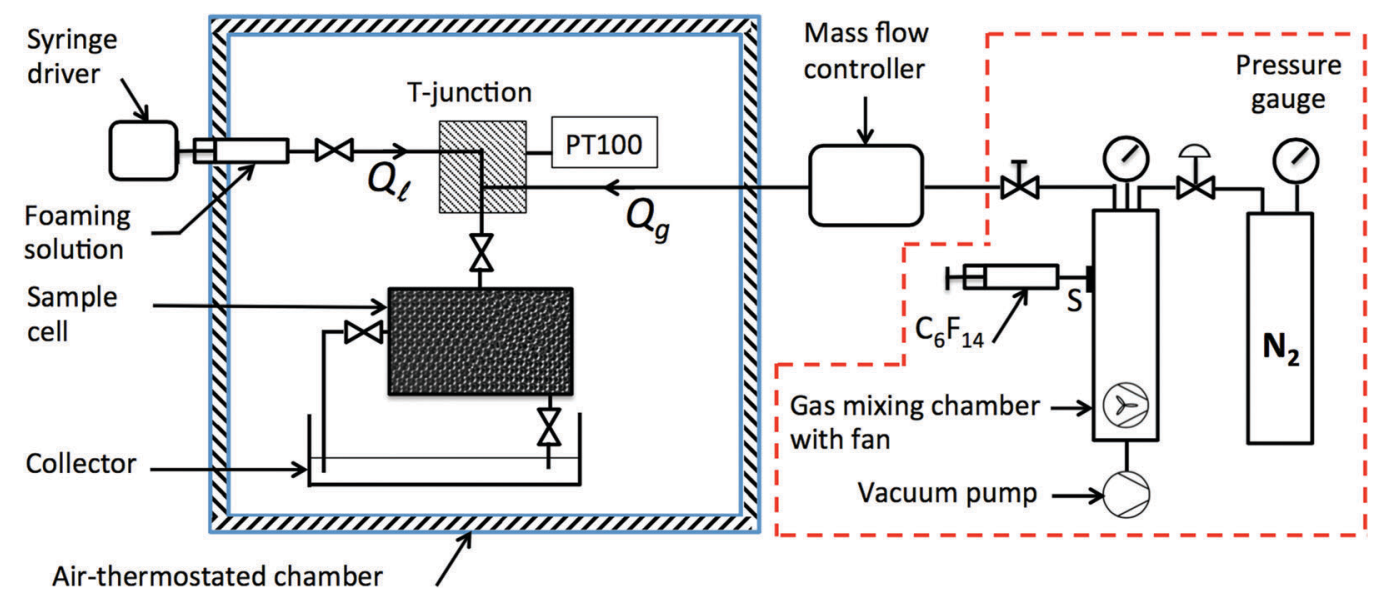

Fig. 1 Schematic diagram of the setup used to prepare gas mixtures (shown in the red dashed frame) and the setup to generate foam in a thermostated chamber. The gas mixture is prepared by injecting a given volume of liquid $\mathrm{C}_{6} \mathrm{~F}_{14}$ through a septum (labelled $\mathrm{S}$ ) into a chamber that has been previously evacuated using a pump. Then the chamber is filled with nitrogen until the total pressure reaches $P_{\mathrm{c}}=2$ bar. Finally, the mixture is homogeneized for 15 minutes using a fan inside the chamber. Monodisperse bubbles are produced using a milli-fluidic T-junction. A syringe pusher injects the foaming solution into the junction with a controlled flow rate $Q_{\ell}$ while the gas mixture is injected at an imposed flow rate $Q_{\mathrm{g}}$ using a mass flow controller. Bubbles are collected in the sample cell, and the gas volume fraction is adjusted by evacuating the appropriate amount of liquid towards the liquid collector. The T-junction, the syringe containing the foaming solution and the sample are all placed in an air-thermostated chamber at $T=44{ }^{\circ} \mathrm{C}$. The temperature is measured close to the T-junction using a PT100 platinum resistance temperature sensor. Once the cell is filled, it is rotated along a horizontal axis using a clinostat (not shown) located inside the thermostated chamber.

the ideal gas constant. The range of $P_{\eta}$ we use, between 1.2 and 180 mbar, remains below the vapor pressure of $\mathrm{C}_{6} \mathrm{~F}_{14}$ which is $\approx 0.3$ bar at $20{ }^{\circ} \mathrm{C}$.

\subsection{Experimental setup and protocol}

The foam samples are generated using the setup schematically represented in Fig. 1. Monodisperse bubbles are produced using a T-junction. For bubble diameters smaller than $1000 \mu \mathrm{m}$, the dimensions of the channel are $800 \mu \mathrm{m}$ width and $400 \mu \mathrm{m}$ depth, while for larger diameters they are $1600 \mu \mathrm{m}$ width and $800 \mu \mathrm{m}$ depth. The foaming solution heated at $50{ }^{\circ} \mathrm{C}$ is injected at a flow rate $Q_{\ell}$ controlled by a syringe pump through one of the inlets of the T-junction. A mass flow controller (Bronkhorst EL-FLOW Select) injects through the second inlet the gas mixture prepared as described in Section 2.3. The T-junction outlet is connected to the inlet of the sample cell made of polycarbonate (internal dimensions: $16 \mathrm{~mm}$ width $\times 27 \mathrm{~mm}$ length $\times 5.3 \mathrm{~mm}$ thickness) where the bubbles are collected within a few minutes, while excess liquid is drained through an outlet toward a liquid collector. The bubbles in contact with the top and bottom faces of the transparent cell can be observed by videomicroscopy.

The average initial diameter of the bubbles, $d=2 R_{\mathrm{o}}$, is controlled by the imposed flow rate ratio $Q_{\ell} / Q_{\mathrm{g}}$ and it can be chosen in the range between $250 \mu \mathrm{m}$ and $1500 \mu \mathrm{m}$. The gas volume fraction value $\Phi$, determined by weight measurements, is $(80 \pm 2) \%$. The typical initial bubble diameter polydispersity calculated as the ratio of the standard deviation to the average diameter is $5-10 \%$.

The syringe containing the foam liquid, the T-junction and the sample cell are all placed in a chamber thermostated at $T=44{ }^{\circ} \mathrm{C}$ by an air flow. At this temperature, the foaming solution is above the gelation point and its viscosity is sufficiently low for operating the T-junction foaming device ( $c f$. Section 2.5).
Once the sample cell is filled with foam at the targeted gas volume fraction, it is disconnected from the T-junction circuit and closed hermetically. This instant defines the initial foam age $t=0$. To homogeneize the liquid content throughout the foam, the cell is then placed on a clinostat inside the thermostated chamber and slowly rotated along an horizontal axis for 20 minutes. After that, the cell is taken out of the thermostated chamber, fixed in a horizontal position and allowed to stand to cool down to room temperature. The foam temperature is measured using a PT100 platinum resistance temperature sensor as a function of aging time $t$. This thermal history is plotted in Fig. 2. Photographs of the top and down surfaces of the sample are regularly taken during the foam aging, from $t=0 \mathrm{~h}$ and typically until $t=16 \mathrm{~h}$ to check that the structure is not modified by drainage before gelation sets in.

\subsection{Rheological characterization}

We use a rheometer (Anton Paar MCR501) equipped with a sand blasted cone-plate geometry $\left(50 \mathrm{~mm}\right.$ diameter, $2^{\circ}$ cone angle) and a Peltier device to control the temperature. The sample is protected against water evaporation by a paraffin oil rim. At the temperature $T=44{ }^{\circ} \mathrm{C}$ where the foam is produced and homogeneized for 20 minutes, the gelatin solutions are liquid. We measure their viscosity at two steady shear rates: $0.5 \mathrm{~s}^{-1}$ and $1 \mathrm{~s}^{-1}$ and find Newtonian response with a viscosity of $7 \mathrm{mPa}$ s for $C_{\text {gel }}=5 \mathrm{wt} \%$ and $20 \mathrm{mPa}$ s for $C_{\text {gel }}=10 \mathrm{wt} \%$.

At a temperature close to $30{ }^{\circ} \mathrm{C}$, gelatin molecules undergo a coil-to-helix transition. It leads to the formation of triple helices that constitute physical cross-links between molecules. ${ }^{33}$ Thus, when the temperature of the gelatin solution is decreased to room temperature $\left(21 \pm 1{ }^{\circ} \mathrm{C}\right)$, the solution is slowly transformed into a physical gel, leading to a progressive increase of 
both the elastic and loss shear moduli $G_{\mathrm{g}}{ }^{\prime}$ and $G_{\mathrm{g}}{ }^{\prime \prime}$. However, these moduli are known to depend on the precise gel thermal history. ${ }^{34}$ Using the rheometer and the cone-plate geometry described above, we apply a sinusuoidal shear strain of $1 \%$ amplitude at a frequency of $1 \mathrm{~Hz}$ and measure the complex shear modulus $G_{\mathrm{g}}{ }^{*}=G_{\mathrm{g}}{ }^{\prime}+\mathrm{i} G_{\mathrm{g}}{ }^{\prime \prime}$, for the same thermal history as in the coarsening experiments described below, up to a sample age of 20 hours. Note that $G_{\mathrm{g}}{ }^{\prime}$ of gelatin is quasi frequency
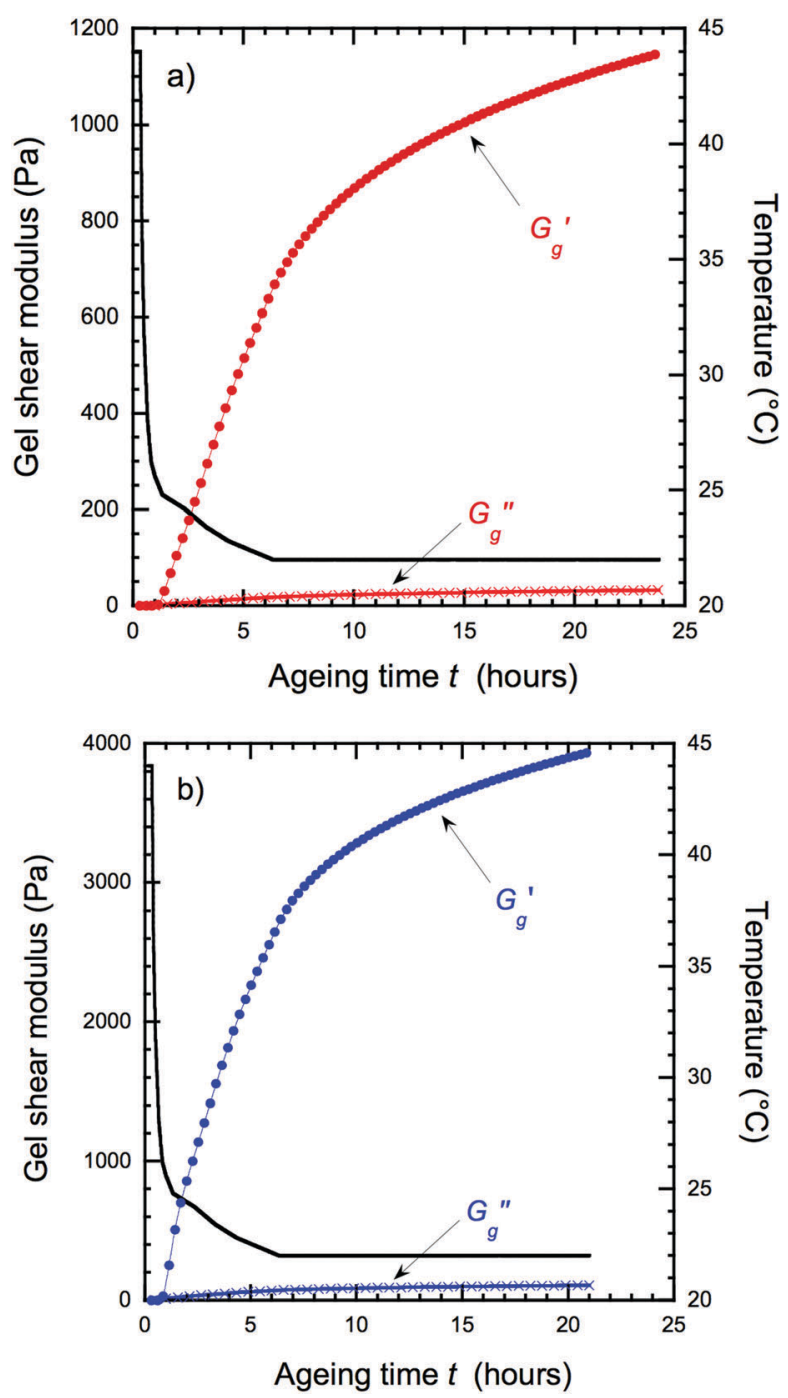

Fig. 2 Thermal and mechanical histories of the gelled foams with Triton X100 C $=0.45$ wt $\%$ and gelatin concentrations: (a) $C_{\text {gel }}=5 \mathrm{wt} \%$, (b) $C_{\text {gel }}=$ $10 \mathrm{wt} \%$. The instant $t=0$ corresponds to the end of the sample production when the cell is filled at the targeted gas volume fraction. During the first 20 minutes, the liquid content of the foam is homogenized at the temperature of the thermostated chamber $\left(44^{\circ} \mathrm{C}\right)$. Then the sample is allowed to cool down to room temperature. The temporal evolution of the foam temperature (see the axis on the right) during aging is plotted as a black continuous line. The storage and loss shear moduli $G_{\mathrm{g}}{ }^{\prime}$ and $G_{\mathrm{g}}{ }^{\prime \prime}$ of the gelatin foaming solutions are measured with a rheometer where they are subjected to the same thermal history as the foam in the coarsening experiments. These bulk gel moduli (see the axis on the left) are plotted versus aging time. Note that on long time scale when the temperature is stabilized to its final value, the storage modulus $G_{\mathrm{g}}{ }^{\prime}$ increases logarithmically with time. independent ${ }^{34}$ in the range $10^{-4}-10 \mathrm{~Hz}$. Using these data, we identify the gelation point as the instant when $G_{\mathrm{g}}{ }^{\prime}=G_{\mathrm{g}}{ }^{\prime \prime}$. Fig. 2 shows the evolutions of $G_{\mathrm{g}}{ }^{\prime}$ and $G_{\mathrm{g}}{ }^{\prime \prime}$ with aging time for both investigated gels. For the less concentrated gelatin solution $\left(C_{\text {gel }}=5 \mathrm{wt} \%\right)$, we observe that, given our protocol, when the temperature decreases below about $26{ }^{\circ} \mathrm{C}$, reaching a foam aging time $t=54 \mathrm{~min}, G_{\mathrm{g}}{ }^{\prime}$ starts to increase ( $c f$. Fig. 2a). For this gel, the gelation point is observed at $t_{\text {gel, } 5}=65$ min when $G_{\mathrm{g}}{ }^{\prime}=G_{\mathrm{g}}{ }^{\prime \prime}$ Pa. Then $G_{\mathrm{g}}{ }^{\prime}$ increases strongly with time. After 15 hours, $G_{\mathrm{g}}{ }^{\prime}=1000 \mathrm{~Pa}$, which is about 30 times larger than $G_{\mathrm{g}}{ }^{\prime \prime}$. For the more concentrated gelatin solution $\left(C_{\text {gel }}=10 \mathrm{wt} \%\right)$, we made similar observations ( $c f$. Fig. 2b): the onset of gelation corresponds to the aging time $t_{\mathrm{gel}, 10}=48 \mathrm{~min} . G_{\mathrm{g}}{ }^{\prime}$ reaches $3700 \mathrm{~Pa}$ after $15 \mathrm{~h}$. At this age, $G_{\mathrm{g}}{ }^{\prime} / G_{\mathrm{g}}{ }^{\prime \prime} \cong 35$. As already well documented, ${ }^{34}$ gelation occurs earlier and the storage modulus grows with increasing gelatin concentration. The mechanical response is predominantly elastic and the storage modulus increases logarithmically on long time scales when the temperature is stabilized at its final value. These observations are consistent with previous measurements of a complex shear modulus of gelatin solutions of similar gelatin concentrations without any surfactant. ${ }^{34,35}$

\section{Mechanisms of foam stabilization by an insoluble gaseous species and gelation of the continuous phase}

As a model system, we consider an ideal monodisperse perfectly ordered foam, constituted of bubbles with an initial radius $R_{\mathrm{o}}$, and a continuous phase made of a soft elastic solid. The gas volume fraction $\Phi$ is a fixed parameter that can take any value between the wet and the dry limits. Each bubble is filled with a mixture of two ideal gases with different solubilities. Following the notations introduced in Section 2.3, we call $\alpha$ the gas that is weakly soluble in the liquid phase, and $\eta$ the insoluble one. Each bubble contains the same fixed number of molecules of the insoluble species $\eta_{\mathrm{o}}$. The total amount of gas in the foam is kept constant as in our experiments. This is a difference between our model and a previous one only valid for dry foams where it is supposed that the bubbles can exchange gas with an infinite reservoir. ${ }^{25}$

To derive a stability criterion for such an ideal foam, we consider that, due to some fluctuation, one anomalous bubble has a radius $R$ slightly different from all the other ones of radius $R_{\mathrm{O}}$. The capillary pressure differences drive a diffusive gas exchange between neighbouring bubbles, and we study how $R$ evolves as a small amount of gas is transferred between this bubble and its neighbours. Since their number (12 for a fcc structure or 14 for a Kelvin one) is large, we neglect the variation of their radii compared to the one of the anomalous bubble. The change of $R$ induces an elastic deformation of the matrix around the anomalous bubble, and an elastic stress that tends to restore the initial unstrained structure. Whether the volume of a bubble increases or decreases, this elastic response always tends to bring the bubble back to its initial volume. 
The matrix elasticity thus stabilizes the foam structure and can counteract the effect of capillary pressure. Moreover the osmotic pressure due to the insoluble trapped gas species tends to establish a uniform concentration of the insoluble species. Since $\eta_{\mathrm{o}}$ is fixed, this osmotic effect works against any variation of the bubble size. It can balance the capillary pressure and stabilize the foam.

We assume that the ensemble of bubbles is dispersed in the bulk liquid containing an initial uniform concentration of the soluble species $\alpha, C_{\infty}$, which corresponds to the saturation at the atmospheric pressure $P_{\mathrm{o}}$. We have $C_{\infty}=H_{\alpha} P_{\mathrm{o}, \alpha}$ where $H_{\alpha}$ is the Henry's law constant as in Section 2.3 and $P_{\mathrm{o}, \alpha}$ is the partial pressure of the species $\alpha$ in the atmosphere. For the soluble species $\alpha$ to be in equilibrium, its chemical potentials in the gas and in the liquid must be equal. This is satisfied when the concentration of the dissolved species $\alpha$ around a bubble of radius $R_{\mathrm{O}}$ is $C_{\mathrm{o}}=C_{\infty} P_{\alpha}\left(R_{\mathrm{o}}\right) / P_{\mathrm{o}, \alpha}$ where $P_{\alpha}\left(R_{\mathrm{o}}\right)$ is the partial pressure of the species $\alpha$ in a bubble. As shown in the Annex, since the solubility of the species $\alpha$ is assumed to be small, the number of moles of gas that must be transferred from one phase to the other to reach $C_{\mathrm{o}}$ is very small compared to the number of moles of $\alpha$ inside a bubble. Thus this small mass transfer does not significantly change the initial bubble size $R_{\mathrm{O}}$ and we neglect it. This feature is a major difference between our model and previous work where coarsening of droplets dispersed in a liquid reservoir was analyzed, depending on its saturation with gas. ${ }^{26}$

First, we consider a spherical bubble, of initial radius $R_{\mathrm{o}}$, immersed in a homogeneous continuous isotropic elastic medium (initially unstrained) which models the surrounding foam, and we determine the relation between the pressure in the bubble $p$ and a small radius variation $R-R_{\mathrm{o}}$. Furthermore, we assume that the elastic deformations remain small so that the matrix elasticity is well described in the linear Hookean limit.

To calculate the displacement field $\mathbf{u}$ at distance $r$ from the bubble center, Navier's equation must be solved. ${ }^{36}$ We consider boundary conditions at the limits of the foam sample in a geometry that can be analyzed analytically: the foam is assumed to have the shape of a spherical shell of inner radius $R_{\mathrm{O}}$ and outer radius $L$, subjected to a pressure $p$ at the inner shell surface. This boundary condition for the radial normal stress $\tau_{\mathrm{rr}}$ thus is written as

$$
\tau_{\mathrm{rr}}=-p \quad \text { at } \quad r=R_{\mathrm{O}}
$$

where $\tau_{i j}$ denotes the Cauchy stress tensor. We assume that the foam is contained in a closed rigid box, so that the displacement at the outer surface of the foam must be equal to zero:

$$
u_{\mathrm{r}}=0 \quad \text { at } \quad r=L
$$

Because of the spherical symmetry, the displacement field is radial and only depends on the distance $r$. Solving Navier's equation yields a radial displacement $u_{\mathrm{r}}$ of the form: ${ }^{36}$ $u_{\mathrm{r}}=A r+B / r^{2}$, where the constants $A$ and $B$ depend on the two Lamé's coefficients of the material $\lambda$ and $\mu$. Using the boundary conditions, eqn (1) and (2), we determine the radial displacement $u_{\mathrm{r}}$ in the shell. In the limit where $L \gg R_{\mathrm{o}}$, the radial displacement at the bubble surface $u_{\mathrm{r}}\left(R_{\mathrm{O}}\right)$ is given to third order in $\left(R_{\mathrm{o}} / L\right)$ by

$$
u_{\mathrm{r}}\left(R_{\mathrm{o}}\right)=R-R_{\mathrm{o}} \cong \frac{p R_{\mathrm{o}}}{4 \mu}-\frac{3 p R_{\mathrm{o}}(\lambda+2 \mu)}{16 \mu^{2}}\left(\frac{R_{\mathrm{o}}}{L}\right)^{3}
$$

The first term is independent of the box size $L$, and the second term rapidly fades away as $\left(1 / L^{3}\right)$. In the case where $\left(R_{\mathrm{O}} / L\right)^{3} \ll(4 / 3) \mu /(\lambda+2 \mu)$, it is negligible and the relation between the pressure inside the bubble and the relative radius variation is linear at order zero in $\left(R_{\mathrm{o}} / L\right)$ :

$$
p=-\tau_{\mathrm{rr}}\left(R_{\mathrm{o}}\right)=4 \mu \frac{R-R_{\mathrm{o}}}{R_{\mathrm{o}}}
$$

The Lamé coefficient $\mu$ is equal to the shear modulus $G$ of the gelled foam matrix: ${ }^{36} \mu=G$. The Lamé coefficient $\lambda$ is related to $G$ and to the Poisson's ratio $\nu$ by $\lambda=2 G \nu /(1-2 \nu)$. Thus the above condition for neglecting the compressibility of the matrix around the bubble is independent of the matrix elasticity $G$ and can be simply written as $\left(R_{\mathrm{O}} / L\right)^{3} \ll 2(1-2 \nu) / 3(1-\nu)$. Eqn (4) is consistent with a previous result derived for small deformations of an isolated bubble contained in a infinite neo-Hookean elastic gel of infinite extent. ${ }^{37}$

Eqn (4) gives the pressure inside a bubble surrounded by a stretched or compressed elastic matrix where the deformation arises from a small variation of the bubble size around $R_{\mathrm{O}}$ corresponding to the reference unstrained state. If the radius $R$ decreases, the matrix around is stretched and exerts on the bubble surface an outward elastic restoring force that tends to bring back the radius to its initial value. An opposite elastic force is induced if $R$ increases. The elastic response of a real gelled foam is more complicated than that of a homogenous medium due to the details of the microstructure such as shape and elasticity of films, Plateau borders and vertices. Therefore, we expect eqn (4) to give the appropriate scaling of the normal stress up to a constant dimensionless prefactor, which we denote as $g$ and we write the normal stress as the following scaling law:

$$
\tau_{\mathrm{rr}}\left(R_{\mathrm{o}}\right)=-4 g G \frac{R-R_{\mathrm{o}}}{R_{\mathrm{o}}}
$$

We now study the stability of an ideal foam where the continuous phase is made of an elastic gel, and we take into account the effect of an insoluble species in the gas mixture. The anomalous bubble mentioned above contains the same number of insoluble molecules $\eta_{\mathrm{o}}$ as all the others since the foam is initially monodisperse. However due to its slightly different size $R$, it has a different partial pressure of the insoluble gas species $P_{\eta}(R)$, given by the ideal gas law:

$$
P_{\eta}(R)=\frac{3 \eta_{\mathrm{o}} k T}{4 \pi R^{3}}=P_{\eta}\left(R_{\mathrm{o}}\right)\left(\frac{R_{\mathrm{o}}}{R}\right)^{3}
$$

where $k$ is the Boltzmann constant. The total gas pressure in the bubble is the sum of $P_{\eta}(R)$ and the partial pressure of the soluble species $P_{\alpha}(R)$ :

$$
P(R)=P_{\alpha}(R)+P_{\eta}(R)
$$


The capillary pressure $P_{\mathrm{c}}(R)$ is defined as the difference between the pressure in the bubble $R$ and the pressure $P_{\mathrm{o}}$ in the surrounding liquid. It is a function of bubble size, gas volume fraction $\Phi$ and surface tension $\gamma$ :

$$
P_{\mathrm{c}}(R)=\frac{\gamma}{R} f(\Phi)
$$

where the $\Phi$-dependent factor $f(\Phi)$ is related to the osmotic pressure of foam. ${ }^{38}$ For the monodisperse fcc foams studied here, the $\Phi$-dependency of this osmotic pressure is known, ${ }^{20}$ and we calculate that the factor $f(\Phi) \approx 2.0$ for $\Phi=0.8$. Since both the interfacial tension and the matrix elasticity contribute to the pressure difference between the bubble and the outside matrix, the partial pressure of the soluble species is

$$
P(R)=P_{\mathrm{o}}+P_{\mathrm{c}}(R)-\tau_{\mathrm{rr}}(R)
$$

Combining eqn (5)-(9) yields the partial pressure of the soluble species in a bubble of radius $R$ :

$$
P_{\alpha}(R)=P_{\mathrm{o}}+\frac{\gamma}{R} f(\Phi)+4 g G \frac{R-R_{\mathrm{o}}}{R_{\mathrm{o}}}-P_{\eta}\left(R_{\mathrm{o}}\right)\left(\frac{R_{\mathrm{o}}}{R}\right)^{3}
$$

Eqn (10) shows how the capillary pressure, the osmotic pressure due to the insoluble trapped species and the elastic stresses compete. To analyze the stability of an anomalous bubble of radius $R$, slightly different from the one of the surrounding bubbles $R_{\mathrm{o}}$, we compare the pressures $P_{\alpha}(R)$ and $P_{\alpha}\left(R_{\mathrm{O}}\right)$ predicted using eqn (10). A pressure imbalance induces a flux of the soluble gas between the anomalous bubble and its neighbours depending on the variation of $P_{\alpha}$ with $R$. If, in the vicinity of $R_{\mathrm{o}}, P_{\alpha}(R)$ is an increasing function of $R$, then a small increase of $R$ above $R_{\mathrm{o}}$ will induce an overpressure $P_{\alpha}(R)-P_{\alpha}\left(R_{\mathrm{o}}\right)>0$ driving an outwards flux of the soluble species. The anomalous bubble will thus shrink. Conversely, a small reduction of $R$ below $R_{\mathrm{O}}$ will lower the pressure in the bubble. Since $P_{\alpha}(R)-P_{\alpha}\left(R_{\mathrm{o}}\right)<0$, it will suck soluble gas from its neighbours and grow. Thus, any bubble size fluctuation around $R_{\mathrm{O}}$ will be damped. Such an ideal foam is stable. Reciprocally, if $P_{\alpha}(R)$ decreases with $R$ close to $R_{\mathrm{o}}$, then any size fluctuation will be amplified and the foam will be unstable. Thus, the criterion for a foam to be stable can be expressed as

$$
\frac{\mathrm{d} P_{\alpha}(R)}{\mathrm{d} R}>0
$$

For a liquid foam, in the absence of elasticity in the continuous phase $(G=0)$, eqn (10) shows that, at fixed initial partial pressure of the insoluble species, $P_{\alpha}(R)$ goes through a maximum for the bubble radius $R_{\mathrm{M}}$ :

$$
R_{\mathrm{M}}=\frac{\gamma f(\Phi)}{3 P_{\eta}\left(R_{\mathrm{o}}\right)}
$$

$R_{\mathrm{M}}$ gives the minimum stable bubble radius as a function of $P_{\eta}\left(R_{\mathrm{o}}\right)$. In practice, $P_{\eta}\left(R_{\mathrm{o}}\right)$ is often the experimental control parameter. Empirical foam stabilization by gas mixtures are qualitatively consistent with this prediction. ${ }^{9,21,27,28}$ Alternatively, using eqn (6), $R_{\mathrm{M}}$ can be expressed as a function of the number of insoluble molecules per bubble, $\eta_{\mathrm{o}}$ :

$$
R_{\mathrm{M}}=\frac{3}{2} \sqrt{\frac{\eta_{\mathrm{o}} k T}{\pi \gamma f(\Phi)}}
$$

According to the stability criterion, eqn (11), foams with bubble radius $R_{\mathrm{O}}<R_{\mathrm{M}}$ will be stable, for a given surface tension and gas volume fraction. Indeed, the osmotic pressure, which tends to establish a uniform concentration of the insoluble species, and scales as $1 / R^{3}$, is the dominant contribution to the pressure $P_{\alpha}(R)$ for small $R$. Conversely, at large bubble radii $R$, the capillary pressure that scales as $1 / R$ and drives coarsening, dominates the pressure $P_{\alpha}(R)$. Thus, at fixed $\eta_{\mathrm{o}}$, foams with small bubbles tend to be more stable than foams with large ones. Note that this effect is consistent with that predicted in dilute emulsions containing an insoluble species trapped in the droplets: ${ }^{25}$ for a fixed number of insoluble species molecules per droplet, the emulsion does not ripen if the droplet radius is small enough for the Laplace pressure to be smaller than the osmotic pressure, ${ }^{25}$ the critical stable droplet radius having a scaling with surface tension and $\eta_{\mathrm{o}}$ similar to that of eqn (13).

We now analyze the foam stability in the case of combined gelation $(G \neq 0)$ and insoluble gas species. To study small variations of $R$ around $R_{\mathrm{o}}$, we set $R=R_{\mathrm{o}}(1+\varepsilon)$ and linearize eqn $(10)$ in $\varepsilon$ :

$$
P_{\alpha}(\varepsilon)=P_{\alpha}\left(R_{\mathrm{o}}\right)+\left(-\frac{\gamma f(\Phi)}{R_{\mathrm{o}}}+4 g G+\frac{9 \eta_{\mathrm{o}} k T}{4 \pi R_{\mathrm{o}}{ }^{3}}\right) \varepsilon
$$

The stability criterion (eqn (11)) corresponds to $\eta_{\mathrm{o}}>\eta_{\mathrm{oM}}$ with

$$
\eta_{\mathrm{oM}}=\frac{4 \pi R_{\mathrm{o}}{ }^{3}}{9 k T}\left(\frac{\gamma f(\Phi)}{R_{\mathrm{o}}}-4 g G\right)
$$

In the absence of any insoluble species in the gas mixture $\left(\eta_{\mathrm{oM}}=0\right)$, coarsening due to capillary pressure differences can then be counterbalanced only by the elasticity of the continuous phase. Eqn (15) shows that the minimum radius for which a bubble can be stable is given by the elasto-capillary radius:

$$
R_{\mathrm{EC}}=\frac{\gamma f(\Phi)}{4 g G}
$$

Note that $R_{\mathrm{EC}}$ can be simply derived using a scaling argument according to which the surface energy $\sim \gamma R_{\mathrm{EC}}{ }^{2}$ must be overcome by the elastic energy $\sim G R_{\mathrm{EC}}{ }^{3}$ to deform a bubble. This radius is similar to the elasto-capillary length characteristics of mechanical problems involving fluid interfaces at sub-millimetric scales: ${ }^{39}$ if the length scale of the interface is smaller than the elasto-capillary length, then the elastic forces are too weak compared to the capillary forces to deform the interface. Eqn (15) can be simply recast in terms of $R_{\mathrm{EC}}$ as

$$
\eta_{\mathrm{oM}}=\frac{16 \pi g G R_{\mathrm{o}}^{2}}{9 k T}\left(R_{\mathrm{EC}}-R_{\mathrm{o}}\right)
$$

According to the stability criterion eqn (11), the ideal foam is stable if $\left(\mathrm{d} P_{\alpha} / \mathrm{d} \varepsilon\right)_{\varepsilon=0}>0$. Thus, at a given modulus $G$ and surface 
a)

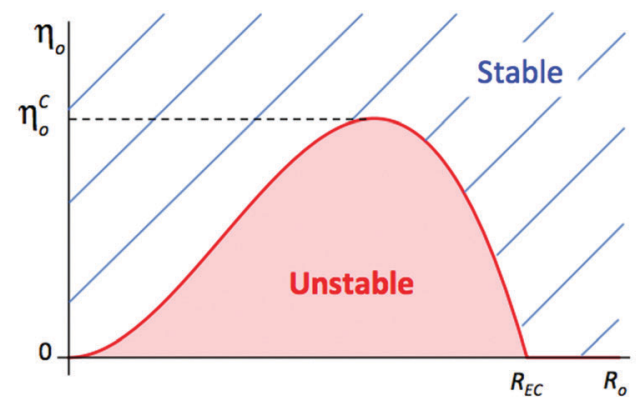

b)

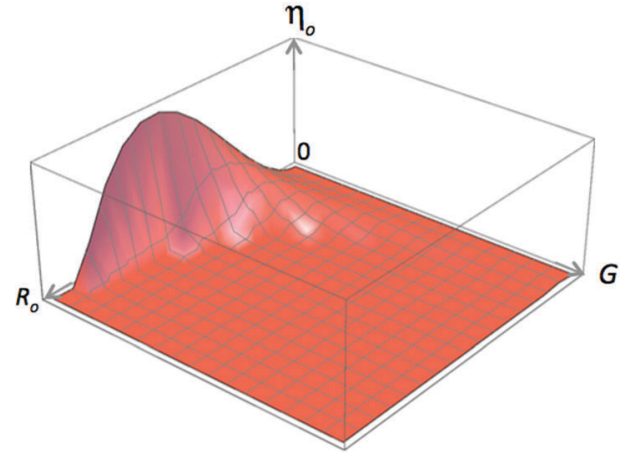

Fig. 3 (a) Schematic stability diagram $\left(R_{0}, \eta_{0}\right)$ of a gelled foam for a given surface tension $\gamma$ and a given elastic modulus of the foam $G$, at a fixed gas volume fraction $\Phi$. The number of insoluble gaseous trapped species, in a bubble of radius $R_{\mathrm{o}}$, is $\eta_{\mathrm{o}}$. The elasto-capillary radius $R_{\mathrm{EC}}$ is defined using eqn (16). In the absence of any insoluble trapped species (when $\eta_{\mathrm{O}}=0$ ), $R_{\mathrm{EC}}$ sets the minimum bubble radius to obtain a stable foam. The continuous curve (in red) represents $\eta_{\circ}$ g given by eqn (17) and (18) for fixed $g, G, \gamma$ and $\Phi$. Its maximum $\eta_{\circ}^{c}$ is defined in the text. According to our stability criterion, the domain of the stable foam corresponds to $\eta_{\mathrm{O}}>\eta_{\mathrm{O}}$. (b) Schematic 3D stability diagram $\left(R_{\mathrm{O}}, G, \eta_{\mathrm{o}}\right)$ of a gelled foam of given surface tension $\gamma$ and gas volume fraction $\Phi$. The stable domain lies above the surface whose equation is given by eqn (17) and (18)

tension $\gamma$, if the bubbles are large enough such that $R_{\mathrm{O}}>R_{\mathrm{EC}}$, this condition is always fulfilled: the gelled foam is stable for any number of insoluble trapped molecules $\eta_{\mathrm{o}}$ as illustrated schematically in Fig. 3a. For small bubbles with $R_{\mathrm{O}}<R_{\mathrm{EC}}$, the stability condition is satisfied only for sufficiently large $\eta_{\mathrm{o}}>\eta_{\mathrm{OM}}$. For fixed modulus $G$ and surface tension $\gamma$, the curve $R_{\mathrm{o}}\left(\eta_{\mathrm{oM}}\right)$ goes through a maximum given by $\eta_{\mathrm{o}}^{\mathrm{c}}=\pi \gamma^{3} f(\Phi)^{3} /\left(243 k T g^{2} G^{2}\right)$. A foam with $\eta_{\mathrm{o}}>\eta_{\mathrm{o}}^{\mathrm{c}}$ will be stable for any bubble radius. We note that the scalings of $R_{\mathrm{EC}}$ and $\eta_{\mathrm{o}}^{\mathrm{c}}$ with $\gamma$ and $G$ are not the same. This means that the knowledge of the elasto-capillary radius $R_{\mathrm{EC}}$ or the ratio $\gamma / G$ is not sufficient to predict the shape of the stable domain in the parameter space $\left(R_{\mathrm{o}}, G, \eta_{\mathrm{o}}\right)$. This constitutes a strong constraint when comparing the data to the predicted stability diagram. In this parameter space, the equation of the surface separating stable and unstable domains is

$$
\eta_{\mathrm{o}}= \begin{cases}0 & \text { if } R_{\mathrm{o}}>R_{\mathrm{EC}} \\ \eta_{\mathrm{oM}} & \text { if } R_{\mathrm{o}}<R_{\mathrm{EC}}\end{cases}
$$

The shape of the surface is shown in Fig. 3b. The unstable domain is located below the surface. As expected, in the limit when the shear modulus $G \rightarrow 0$ (or $R_{\mathrm{EC}} \rightarrow \infty$ ), the frontier of stability predicted by eqn (15) is identical to that predicted by eqn (13) for liquid foams with the parameters $\left(R_{\mathrm{o}}\right.$ and $\left.\eta_{\mathrm{o}}\right)$.

\section{Results}

\subsection{Liquid foams}

As a reference case, we first study the stability of liquid foams without gelatin, depending on the initial bubble diameter $d=2 R_{\mathrm{O}}$ and the initial partial pressure $P_{\eta}=P_{\eta}\left(R_{\mathrm{o}}\right)$ of the insoluble gas species $\mathrm{C}_{6} \mathrm{~F}_{14}$. Initially the foam structures are ordered with large crystalline domains at the surface as shown in Fig. 4 and 5.

In the absence of $\mathrm{C}_{6} \mathrm{~F}_{14}$, for all of the investigated bubble sizes, we observe foam destabilization by coarsening after typically $\sim 1$ hour of aging: bubbles much larger than the initial size as well as structural disorder appear, as shown in Fig. 4.

\section{Liquid foams without gelatin with pure $\mathrm{N}_{2}$ gas}
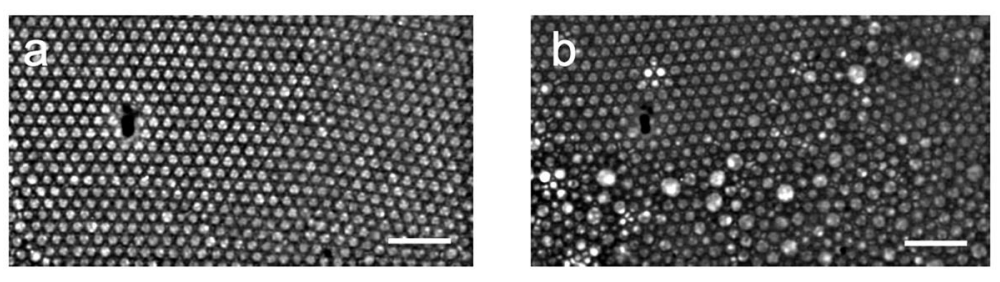

$0 \min$

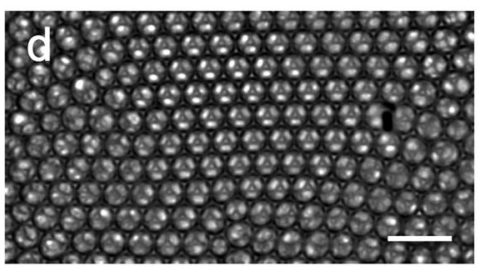

$0 \min$
$20 \mathrm{~min}$

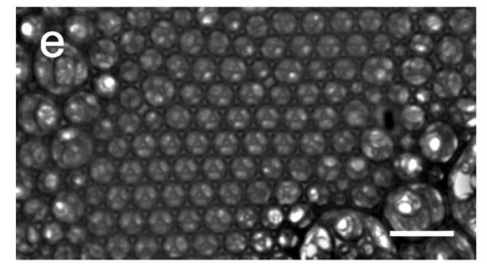

$1 \mathrm{~h}$
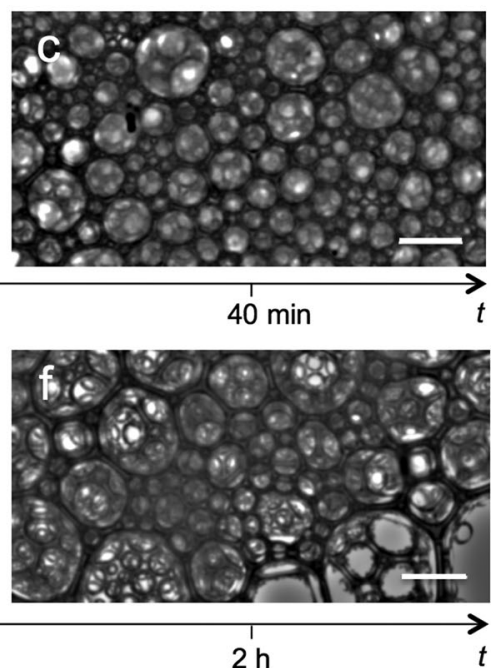

Fig. 4 Photographs of two liquid foams $(\Phi=79 \%)$ without gelatin and with pure $\mathrm{N}_{2}$ gas, at different ages as indicated, for two initial bubble diameters: $(\mathrm{a}-\mathrm{c}) d=325 \mu \mathrm{m}$; $(\mathrm{d}-\mathrm{f}) d=994 \mu \mathrm{m}$. For a given sample, the views are taken at the same place. The scale bars are $2 \mathrm{~mm}$. 
For a given bubble size the aging time where coarsening becomes visible varies slightly from one sample to another due to the differences in their structures: crystalline domain walls and structural defects that act as nucleation sites for coarsening are not identical for different samples. Thus, we see that 1 hour is a typical time scale of coarsening due to nitrogen diffusion for our liquid foam samples in the absence of $\mathrm{C}_{6} \mathrm{~F}_{14}$. Videomicroscopy observations of the top and the bottom surfaces of the samples did not show any evidence of drainage or coalescence on this time scale of observation. We choose the following stability criterion: as long as clusters of more than 50 bubbles with the initial size remain after 1 hour aging, we consider that a foam with such given $d$ and $P_{\eta}$ is a stable one.

The kinetics of gas transport through a liquid film between two bubbles is controlled by the product $D H$ where $D$ is the gas molecular diffusion coefficient in water and $H$ is Henry's law constant. ${ }^{9}$ The ratio of the diffusion coefficients ${ }^{40}$ of $\mathrm{C}_{6} \mathrm{~F}_{14}$ to that of nitrogen, $D_{\eta} / D_{\alpha}$ is $\approx 0.3$. Thus, we have $H_{\eta} D_{\eta} /\left(H_{\alpha} D_{\alpha}\right) \approx$ $2 \times 10^{-5}$. Therefore the effect of the finite solubility of $\mathrm{C}_{6} \mathrm{~F}_{14}$ should have an impact on coarsening only on timescales several orders of magnitude larger than that of nitrogen, and we do not expect to observe it. Moreover effects due to the solubility of $\mathrm{CO}_{2}$ would be expected on timescales of the order of a few tenths of seconds just after foam production, which is several orders of magnitude shorter than the time scales of our experiments. ${ }^{41}$

To characterize the impact of insoluble species on coarsening, we observe the structure of foams with different bubble sizes and partial pressures of $\mathrm{C}_{6} \mathrm{~F}_{14}$. For instance, the foam in Fig. 5(a-c) is an "unstable" one since after $1 \mathrm{~h}$ of aging its structure is modified: the bubble sizes become polydisperse. After $2 \mathrm{~h}$ of aging, only small bubble clusters with the initial bubble diameter remain. The liquid foam as in Fig. $5(\mathrm{~d}-\mathrm{f})$ is a "stable" one. Its structure is not modified after $1 \mathrm{~h}$ of aging. Fig. 5 also shows the impact of varying the bubble diameter $d$ at constant partial pressure. For $P_{\eta}=0.12$ bar and $d \approx 300 \mu \mathrm{m}$, Fig. 5(d-f) shows that after 16 hours of aging, some bubbles much larger than $d$ are present. However, large clusters of more than 50 bubbles with the initial diameter are still present. Therefore, we consider this foam as "stable". For the foam with larger initial bubbles $d \approx 1000 \mu \mathrm{m}$ (Fig. 5g-i), structural changes are even smaller than for $d=300 \mu \mathrm{m}$ over the same laps of time.

We analyze the bubble size distributions on photographs taken at the aging times $t=0 \mathrm{~h}, t=8 \mathrm{~h}$ and $t=16 \mathrm{~h}$. Inspection of the photographs taken from above and below the cell show that the bubble sizes and the liquid fraction are identical on both sides of the cell ( $c f$. Fig. 6). Based on a population of

\section{Liquid foams without gelatin}

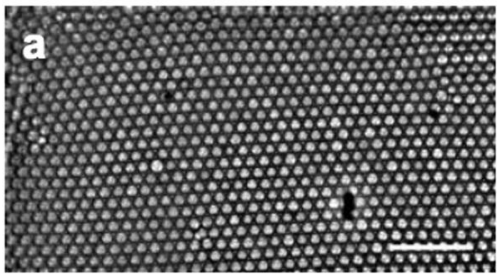

$20 \mathrm{~min}$

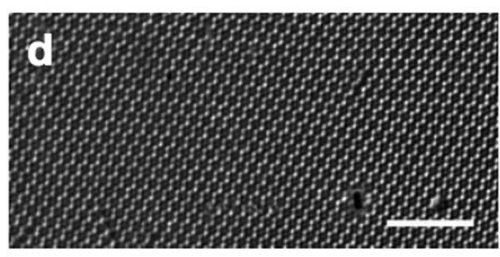

$20 \mathrm{~min}$

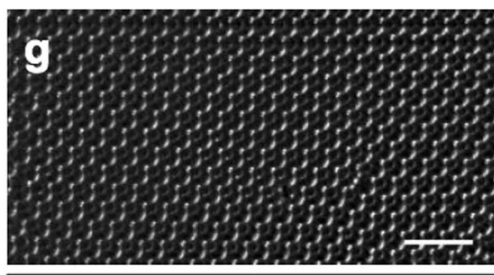

$20 \mathrm{~min}$

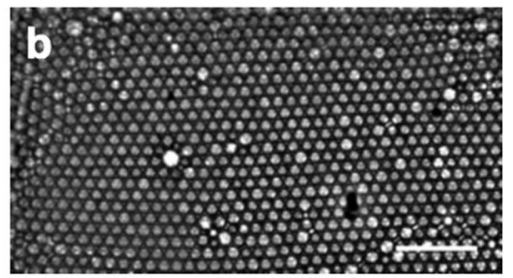

$1 \mathrm{~h}$

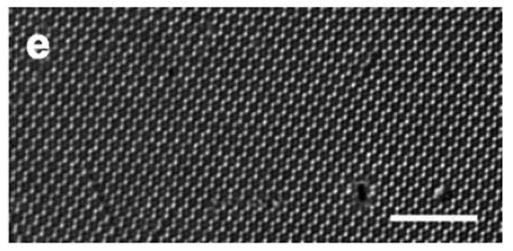

$1 \mathrm{~h}$

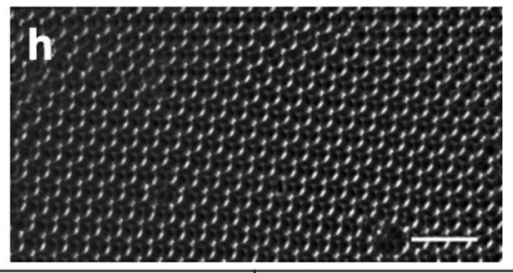

$1 \mathrm{~h}$
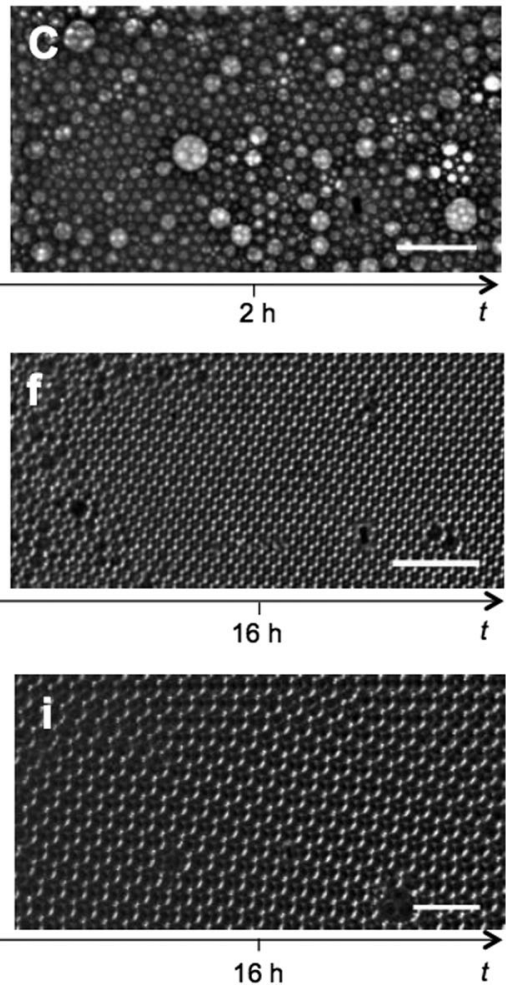

Fig. 5 Photographs of three liquid foams without gelatin, at age $t=20 \mathrm{~min}$ (just after homogenization, cf. Section 2.4) and at later ages, with different initial bubble diameters and partial pressures $P_{\eta}$ of $\mathrm{C}_{6} \mathrm{~F}_{14}$ : (a-c) $d=336 \mu \mathrm{m}, P_{\eta}=0.0065$ bar; $(\mathrm{d}-\mathrm{f}) d=316 \mu \mathrm{m}, P_{\eta}=0.12 \mathrm{bar} ;(\mathrm{g}-\mathrm{i}) d=1000 \mu \mathrm{m}, P_{\eta}=0.12$ bar. All the foams have the same gas volume fraction $\phi=79 \%$. The structure of the foam in photos $a, b$, and c considerably evolves in 1-2 hours: it is an unstable foam that coarsens on this time scale due to nitrogen diffusion between bubbles. In photos $d, e$, and $f$, the foam is stable since large bubble clusters with the initial size $d$ can be seen. On this time scale coarsening due to nitrogen diffusion is inhibited by the presence of the insoluble $\mathrm{C}_{6} \mathrm{~F}_{14}$ molecules. In photos $\mathrm{g}, \mathrm{h}$, and $\mathrm{i}$, the foam is stable. Only a few bubbles with different sizes appear. For a given sample, the views are taken at the same place. All scale bars represent $3 \mathrm{~mm}$. 

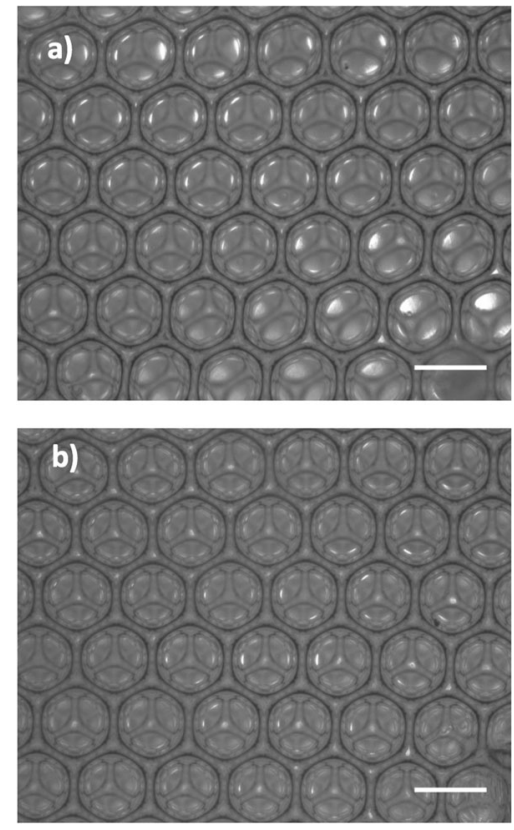

Fig. 6 Photographs taken on top (a) and bottom (b) of the cell showing that the foam structure is similar after 16 hours aging, in the absence of gelatin (bubble diameter $d=1000 \mu \mathrm{m}, P_{\eta}=0.12 \mathrm{bar}, \Phi=0.80$ ). The scale bars represent $1 \mathrm{~mm}$.

500 bubbles at each age, we measure the normalized probability density of the bubble diameters, the average bubble diameters and the standard deviations. Fig. 7 shows the evolution of the average diameter with foam age for three different initial bubble sizes, at a given partial pressure $P_{\eta}$. At this pressure, the average diameter slightly increases for the two smallest bubble sizes $(d \approx 300 \mu \mathrm{m}$ or $600 \mu \mathrm{m})$ while it remains constant for the larger ones $d \approx 1000 \mu \mathrm{m}$. At the same time, there is a net increase of the standard deviation for the smallest diameters. This is consistent with the evolution of the bubble size distributions shown in Fig. 8. After $16 \mathrm{~h}$ of aging, in the case $d \approx 300 \mu \mathrm{m}$ (Fig. 8a), the proportion of large bubbles increases, building up a tail in

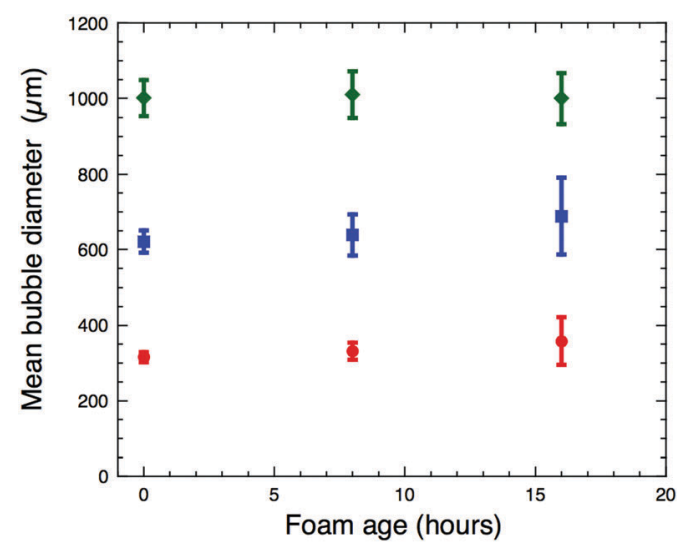

Fig. 7 Evolution of the mean bubble diameter as a function of foam age for liquid foams without gelatin, having different initial bubble diameters $d$ : ( ) $320 \mu \mathrm{m},(\boldsymbol{\square}) 620 \mu \mathrm{m}$, and ( ) $1000 \mu \mathrm{m}$. The bars represent the standard deviations. The $\mathrm{C}_{6} \mathrm{~F}_{14}$ partial pressure is $P_{\eta}=0.12$ bar, as in Fig. 5 .
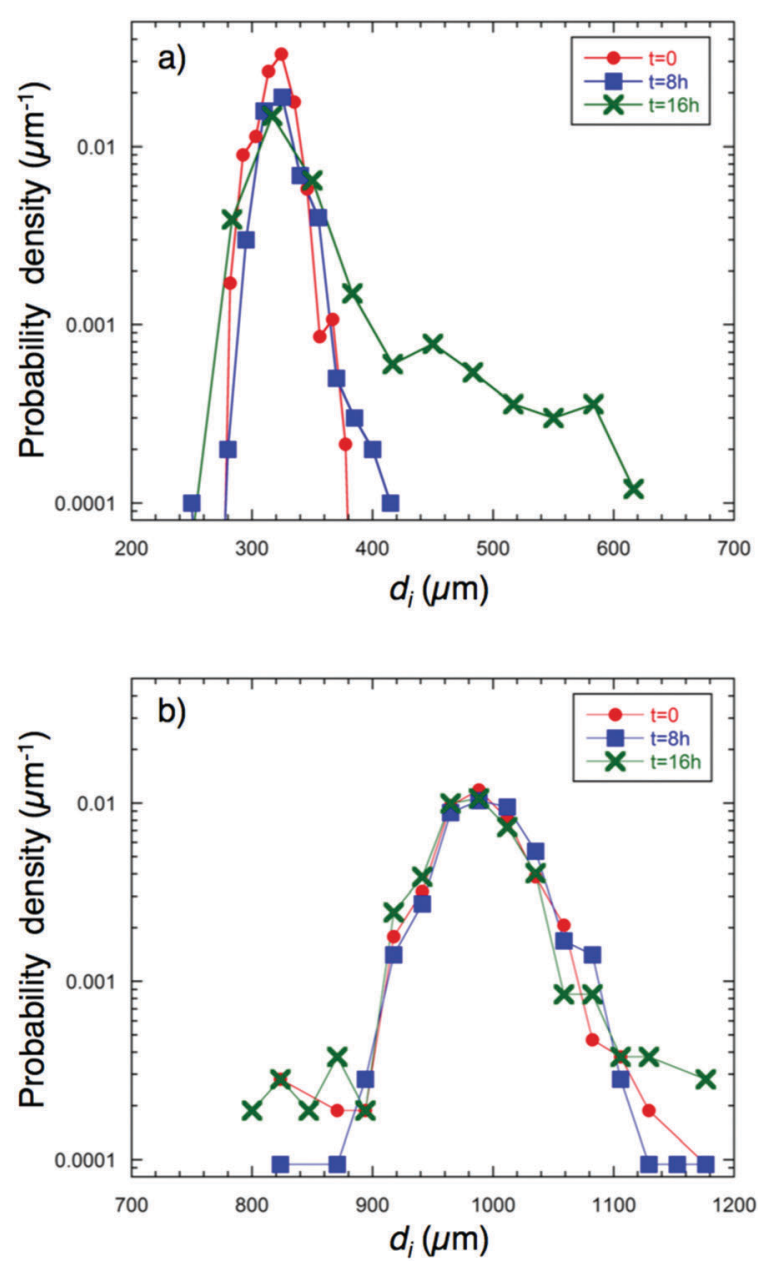

Fig. 8 Probability density of the bubble diameter for liquid foam without gelatin for different foam ages as labeled. The initial bubble diameters are: (a) $d=320 \mu \mathrm{m}$, and (b) $d=1000 \mu \mathrm{m}$, with $P_{\eta}=0.12$ bar (same data as in Fig. 7). The lines are guides to the eye.

the distribution, while no significant evolution of the distribution is detected in the case $d \approx 1000 \mu \mathrm{m}$ (Fig. 8b).

To summarize, by systematically varying the initial bubble size $d$ and the partial pressure $P_{\eta}$ of $\mathrm{C}_{6} \mathrm{~F}_{14}$, we observe that the smallest bubble size for which stability can be achieved decreases as $P_{\eta}$ increases. This is consistent with previous observations. ${ }^{9,21,27,28}$ In Section 5.1, we compare the observed domain of the stability of such foams to that predicted above for ideal foams ( $c f$. Section 3).

\subsection{Gelled foams}

We investigate the aging of gelled foams as a function of the bubble size $d=2 R_{\mathrm{o}}$, the gelatin concentration $C_{\text {gel }}$ in the foaming solution and the partial pressure $P_{\eta}=P_{\eta}\left(R_{\mathrm{o}}\right)$ of the insoluble species $\mathrm{C}_{6} \mathrm{~F}_{14}$ in the gas mixture. Initially the foam structures are ordered with large crystalline domains as for the liquid foams. We recall that during the first 20 minutes of aging, the sample is still placed inside the thermostated chamber at $44{ }^{\circ} \mathrm{C}$ and the continuous phase does not yet exhibit a measurable shear modulus ( $c f$. Fig. 2). Moreover, the onset of 
gelation corresponds to the instants $t_{\mathrm{gel}, 5}=65 \mathrm{~min}$ and $t_{\mathrm{gel}, 10}=$ $48 \mathrm{~min}$ for the respective gelatin concentrations $C_{\text {gel }}=5 \mathrm{wt} \%$ and $C_{\text {gel }}=10 \mathrm{wt} \%$ ( $c f$. Fig. 2). Thus, it is only after these aging durations that elastic forces can be generated by the gelation of the continuous phase and potentially counteract bubble deformation induced by gas exchanges between neighbouring bubbles.

Our observations show that extremely stable foams can be obtained if the bubble size $d$, the partial pressure $P_{\eta}$ or the gelatin concentration $C_{\text {gel }}$ are sufficiently large. On the contrary, if one of these parameters is too small, coarsening is observed. Similarly to the case of liquid foams, crystalline domain walls and structural defects act as nucleation sites for coarsening in gelled foams. We determine the age $t_{\max }$ when we observe the last cluster of at least 50 bubbles with the initial diameter $d$, and we consider that a foam with such $d, P_{\eta}$ and the storage modulus of the continuous phase evaluated at $t_{\max }$ using the data in Fig. 2 is "stable".

As an illustration, Fig. 9 shows the evolution of gelled foams with the same $P_{\eta}=0.06$ bar, but different $d$ or $C_{\text {gel }}$. With $C_{\text {gel }}=$ $5 \mathrm{wt} \%$ and $d=330 \mu \mathrm{m}$ (Fig. 9a and b), we observe that at $12 \mathrm{~h}$, the foam structure exhibits clusters with an initial bubble size $d$ surrounded by regions with heterogeneous bubble sizes. Inspection of the photographs at ages beyond $12 \mathrm{~h}$ reveals that the clusters become smaller than about 50 bubbles. Thus, in this case, we have $t_{\max }=12 \mathrm{~h}$. At this instant, the modulus of the gel is $G_{\mathrm{g}}{ }^{\prime}=930 \mathrm{~Pa}(c f$. Fig. 2a), and we deduce that a foam with the set of parameters $d=330 \mu \mathrm{m}, P_{\eta}=0.06 \mathrm{bar}$ and $G_{\mathrm{g}}{ }^{\prime}=930 \mathrm{~Pa}$ is stable. With the same gelatin concentration and larger bubbles $d=1026 \mu \mathrm{m}$, we observe in Fig. 9c and $d$ that the

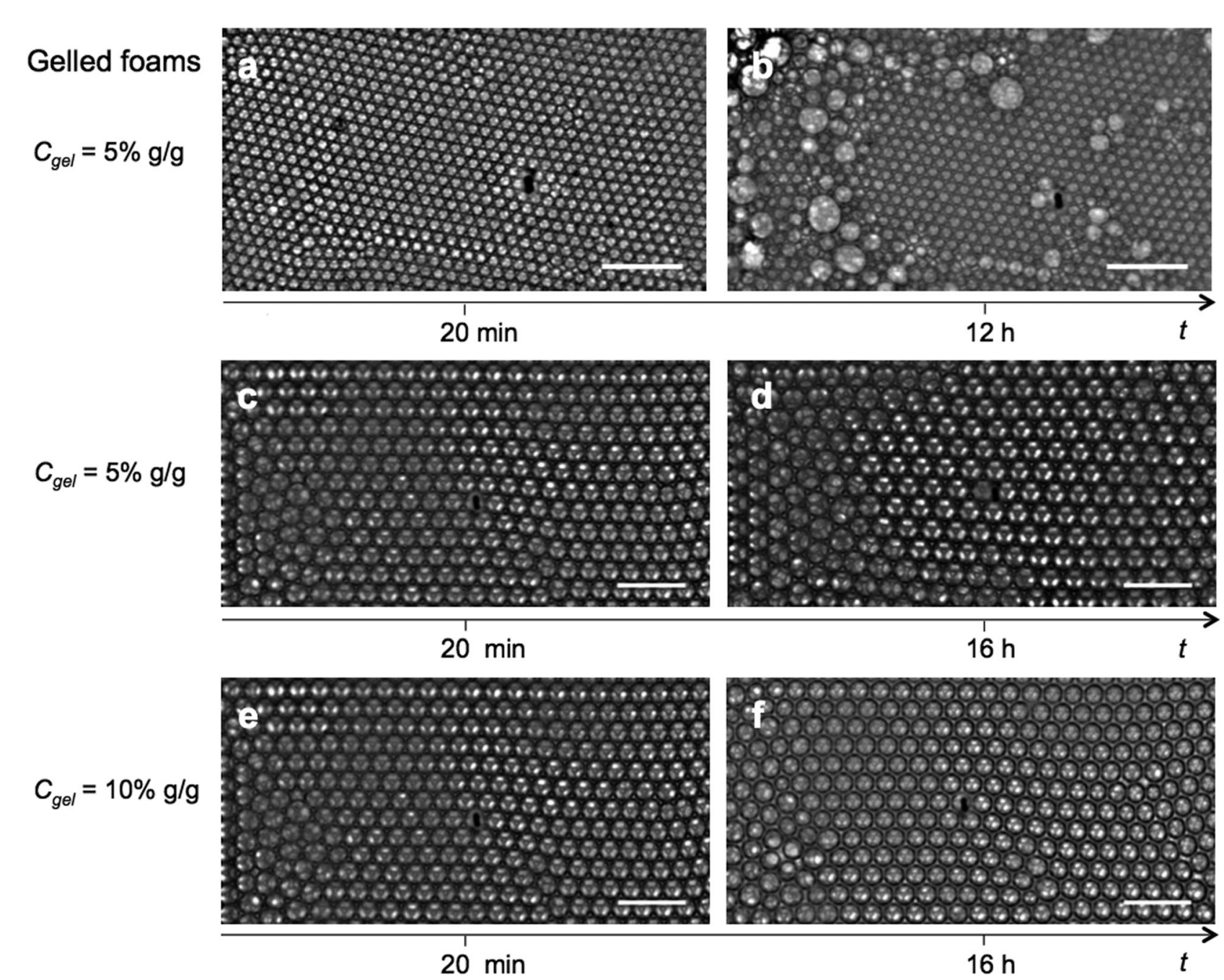

Fig. 9 Photographs of three monodisperse gelled foams with the same partial pressure of $\mathrm{C}_{6} \mathrm{~F}_{14}, P_{\eta}=0.06$ bar, at age $t=20$ min (just after homogeneization) and at later ages as indicated, with different gelatin concentrations and initial bubble diameters: (a and b) $C_{\text {gel }}=5 \mathrm{wt} \%, d=330 \mu \mathrm{m}$; (c and d) $C_{\text {gel }}=5 \mathrm{wt} \%, d=1030 \mu \mathrm{m}$; (e and f) $C_{\text {gel }}=10 \mathrm{wt} \%, d=890 \mu \mathrm{m}$. All foams have the same gas volume fraction $\Phi=79 \%$. For the foam in (a and b), bubble clusters of at least 50 bubbles perdure up to an age of $t_{\max }=12 \mathrm{~h}$. Beyond this age the clusters become smaller. $t_{\max }$ is the age limit of foam stabilization after which the coarsening due to nitrogen diffusion dominates over the gel elasticity. The foams in (c and d) and (e and f) are stable up to at least $t_{\max }=16 \mathrm{~h}$. They are stabilized against coarsening by the gel elasticity. The storage moduli of the foams are evaluated at $t_{\max }$ using the measured values of the storage modulus of the continuous phase shown in Fig. 2 and eqn (19). For a given sample, the views are taken at the same place. The scale bars represent $3 \mathrm{~mm}$. 
foam is stable up to $t_{\max }=16 \mathrm{~h}$. At this age, we have $G_{\mathrm{g}}{ }^{\prime}=1000 \mathrm{~Pa}$. Consistently, increasing the gelatin concentration to $C_{\text {gel }}=10 \mathrm{wt} \%$ while keeping a similar bubble size ( $c f$. Fig. 9e and f) yields a foam that is stable up to $16 \mathrm{~h}$. At this age, $G_{\mathrm{g}}{ }^{\prime}=3700 \mathrm{~Pa}$ ( $c f$. Fig. 2b). A fortiori, these three foams are also stable at $t=1 \mathrm{~h}<t_{\mathrm{gel}, 5}$, i.e. during the period preceding gelation when $G_{\mathrm{g}}{ }^{\prime}=0 \mathrm{~Pa}$, which is consistent with our observations of liquid foams ( $c f$. Section 4.1).

Fig. 10 shows the evolution of foams with similar bubble sizes and gelatin concentrations as in Fig. 9 but with a larger partial pressure of $\mathrm{C}_{6} \mathrm{~F}_{14}, P_{\eta}=0.12$ bar. We see that the foam with $C_{\text {gel }}=5 \mathrm{wt} \%$ and the smallest bubble size $d=330 \mu \mathrm{m}$ is now stable up to $t_{\max }=16 \mathrm{~h}$. We compare the bubble size distributions of this foam at the ages $t=0 \mathrm{~h}$ and $t=16 \mathrm{~h}$. We measure the normalized probability density of the bubble diameter based on a population of 500 bubbles at each age. The average bubble diameter and the standard deviation are constant over time and we do not detect any significant evolution of the distribution as shown in Fig. 11. Moreover, we expect foams with the same $P_{\eta}$ and a larger bubble size or gelatin concentration to be stable up to at least $16 \mathrm{~h}$. This is indeed the case as illustrated by the comparison between Fig. 10a-f.

Furthermore, we observed that gelled foams with a sufficiently large bubble size can be stable beyond $16 \mathrm{~h}$ if the $\mathrm{C}_{6} \mathrm{~F}_{14}$ partial pressure is large enough to stabilize the foam until the gelation starts. For $C_{\text {gel }}=5 \mathrm{wt} \%$, no structural changes are observed during at least the next 4 days if the bubble diameter exceeds a characteristic value $d_{5} \approx 1000 \mu \mathrm{m}$. For instance, structures such as those in Fig. 9c, d and 10c, d indeed perdure for several days. We made similar observations for $C_{\text {gel }}=10 \mathrm{wt} \%$ and found that foams with $d$ larger than $d_{10} \approx 300 \mu \mathrm{m}$ are stable for several days.

To summarize, there are three parameters that govern the stabilization of gelled foams: the bubble diameter, the partial pressure of the insoluble species and the storage modulus of

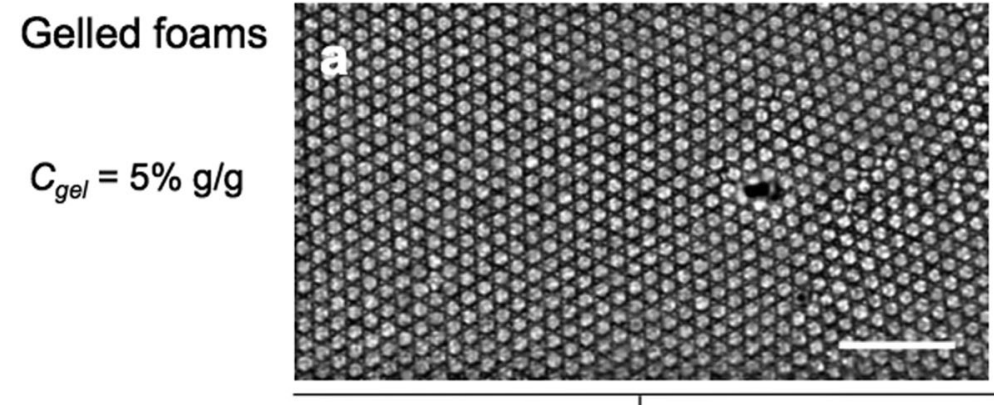

$20 \min$

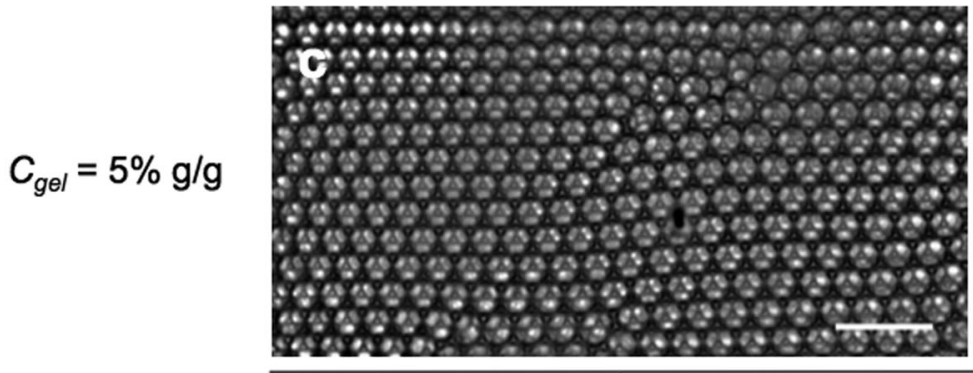

$20 \mathrm{~min}$

$C_{g e l}=10 \% \mathrm{~g} / \mathrm{g}$

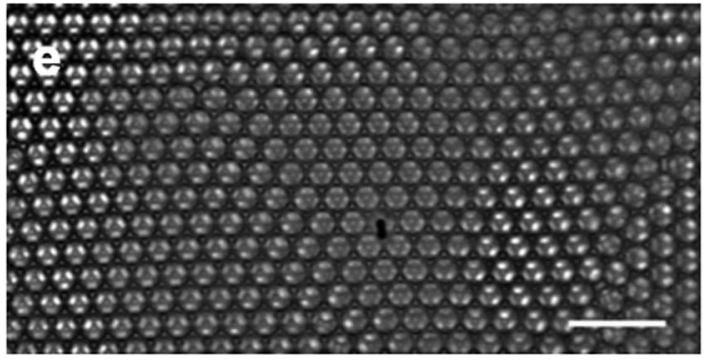

$20 \min$

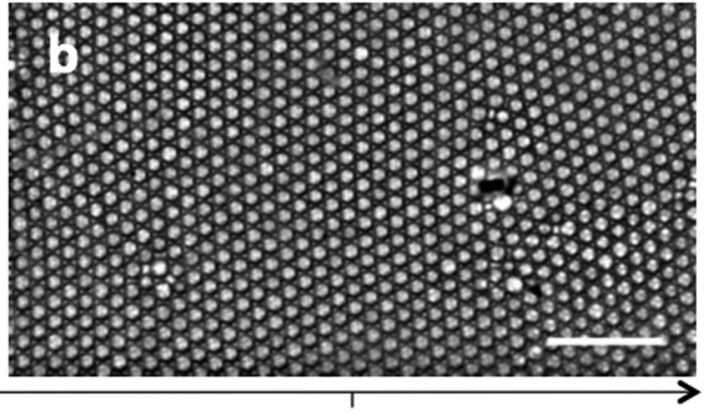

$16 \mathrm{~h}$

$t$

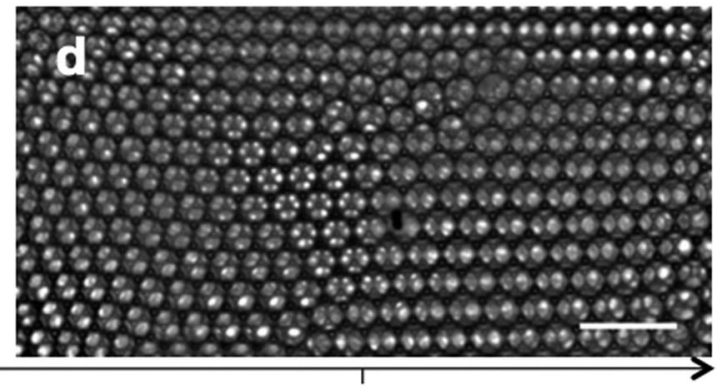

$16 \mathrm{~h}$

$t$

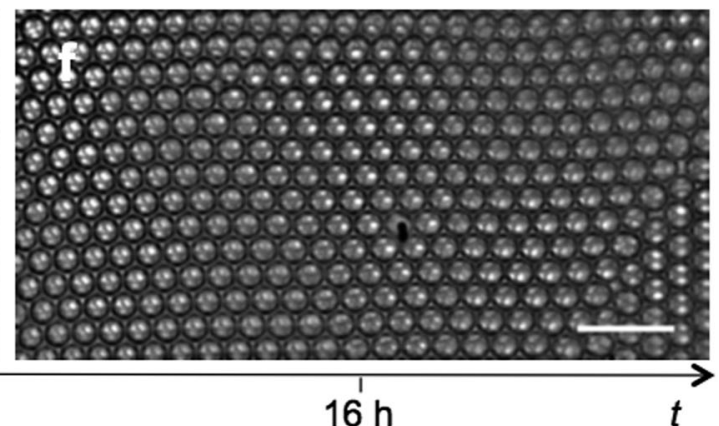

Fig. 10 Photographs of three monodisperse gelled foams, with the same partial pressure of $\mathrm{C}_{6} \mathrm{~F}_{14}, P_{\eta}=0.12$ bar at age $t=20$ min (just after homogeneization) that are stable up to at least $t_{\max }=16 \mathrm{~h}$, with different gelatin concentrations and initial bubble diameters: $(a$ and $\mathrm{b}) \mathrm{C}_{\mathrm{gel}}=5 \mathrm{wt} \%$, $d=330 \mu \mathrm{m}$; (c and d) $C_{\mathrm{gel}}=5 \mathrm{wt} \%, d=980 \mu \mathrm{m}$; (e and f) $C_{\mathrm{gel}}=10 \mathrm{wt} \%, d=870 \mu \mathrm{m}$. All of the foams have the same gas volume fraction $\Phi=79 \%$. The storage modulus of the foam is evaluated at $t_{\max }$ using the measured values of the storage modulus of the continuous phase shown in Fig. 2 and eqn (19). For a given sample, the views are taken at the same place. All scale bars represent $3 \mathrm{~mm}$. 


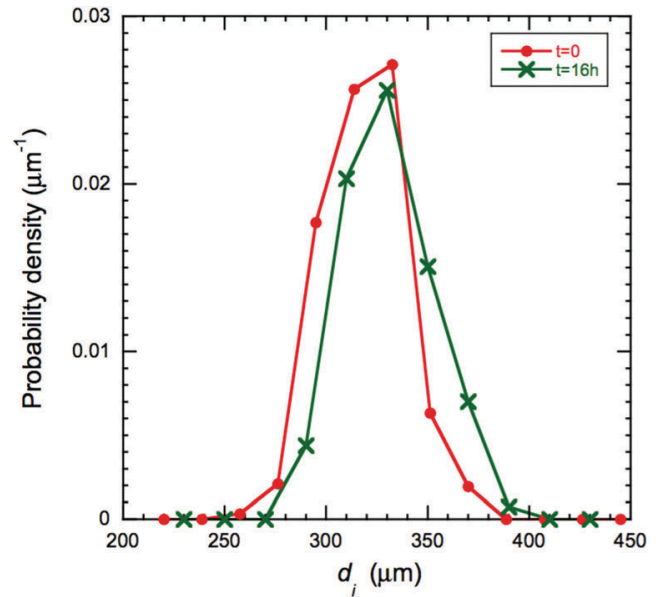

Fig. 11 Probability density of the bubble diameter for a gelled foam with $C_{\text {gel }}=5$ wt $\%$ and $\mathrm{C}_{6} \mathrm{~F}_{14}$ partial pressure $P_{\eta}=0.12$ bar, for two ages as labelled. The initial bubble diameter is $d=330 \mu \mathrm{m}$ with standard deviation equal to $20 \mu \mathrm{m}$. After $16 \mathrm{~h}$, the diameter is $340 \mu \mathrm{m}$ and the standard deviation is $21 \mu \mathrm{m}$ (Data from Fig. 10a and b). The distributions are determined from a sampling of 500 bubbles at each age. No tail appears in the distribution indicating that the gelled foam is stable. The lines are guides to the eyes.

the continuous phase. In Section 5.2, we compare our results to the stability diagram predicted for ideal foams ( $c f$. Section 3 ).

\section{Discussion}

\subsection{Liquid foams}

In Fig. 12, we plot the initial radius $R_{\mathrm{o}}$ of the bubbles as a function of the quantity $\sqrt{\eta_{\mathrm{o}}}$ to compare our data for stable and unstable foams to the stability diagram predicted for ideal foams. We recall that the frontier between stable and unstable ideal foams is predicted by $R_{\mathrm{M}}$ (eqn (13)). Our data show that the observed stability frontier can indeed be described by a straight line in qualitative agreement with eqn (13), of the form: $R^{*}=C R_{\mathrm{M}}$ with $C \approx 0.29$. Thus, the coefficient $C$ is 3.3 times smaller than that predicted. The inset of Fig. 12 shows the same data where the initial bubble radius $R_{\mathrm{O}}$ is plotted as a function of the initial partial pressure $P_{\eta}$ of the insoluble $\mathrm{C}_{6} \mathrm{~F}_{14}$ species. The observed line separating the stable and unstable domains is consistent with the frontier observed in the $\left(R_{\mathrm{o}}, \sqrt{\eta_{\mathrm{o}}}\right)$ diagram. The consistency between the observed scaling and the predicted one shows that the parameters that control the stability of the foam are indeed $R_{\mathrm{o}}$ and $\sqrt{\eta_{\mathrm{o}}}$ or $P_{\eta}$. In addition our results show that the observed stability domain is less extended towards large bubbles than expected for an ideal foam. We presume that the discrepancy is due to the disorder of the real foams. Our samples exhibit a small bubble size polydispersity as illustrated by the measured size distributions (cf. Fig. 8 and 11) as well as topological disorder due to structural packing defects such as grain boundaries ( $c f$. Fig. 5, 9 and 10). In such a disordered region, the bubble surfaces are distorted and the bubbles have a surface curvature different from that of a bubble in the ordered packing, and therefore a

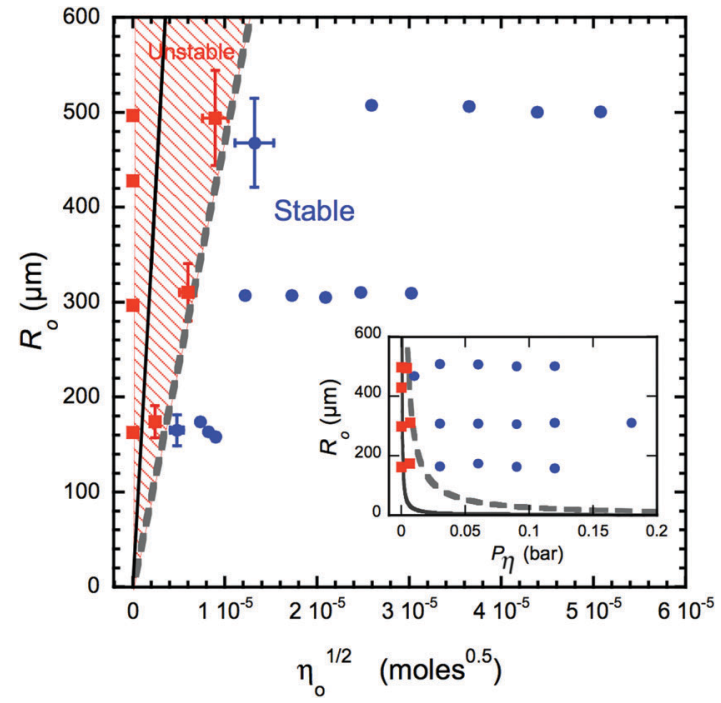

Fig. 12 Diagram showing stable $(0)$ or unstable (red $\mathbf{\square}$ ) liquid foams without gelatin depending on their initial bubble radius $R_{\circ}$ and number $\eta_{\circ}$ of moles of insoluble $\mathrm{C}_{6} \mathrm{~F}_{14}$ gas per bubble. The stability of the foams is observed after one hour of aging. The width of the error bars is estimated from the standard deviation of the bubble radius. The continuous straight line corresponds to the stability frontier predicted for an ideal foam by $R_{\mathrm{M}}$ (eqn (13)) with $\Phi=0.8, f(\Phi)=2.0, \gamma=0.0313 \mathrm{~N} \mathrm{~m}^{-1}, T=295 \mathrm{~K}$. The dashed straight line represents the observed frontier: $R^{\star}=C R_{\mathrm{M}}$ with $C \approx 0.3$. Inset: Same data (with the same symbols) plotted as a function of the initial partial pressure of $\mathrm{C}_{6} \mathrm{~F}_{14}$. The continuous line represents $R_{\mathrm{M}}$ (eqn (12)) whereas the dashed line corresponds to the radius $R^{\star}=\gamma f(\Phi) /\left(3 C^{2} P_{\eta}\right)$ with $C=0.29$. The parameters are the same as in the main graph.

different capillary pressure. In the extreme case of a disordered structure, previous experiments have shown that the local osmotic pressure can vary by a factor of 5 in a given packing, ${ }^{42}$ leading to local fluctuations of capillary pressure. This is a strong indication that, due to structural effects, the stability limit can become fuzzy. A quantitative study of the impact of disorder on the limit of stability deserves more theoretical work that is beyond the scope of the present work.

\subsection{Gelled foams}

To compare our prediction, eqn (17), of the stability domain of ideal gelled foams to our data, we need to estimate the shear modulus of the studied foams $G$. Previous acoustic measurements on similar ordered gelatin foams show that they behave as solid foams with closed cells, and that in this range of gas volume fraction $\Phi$, the contribution of the films to the elastic response is negligible compared to that of the Plateau borders. ${ }^{43}$ The foam storage shear modulus $G$ is related to the storage shear modulus of the continuous gelled phase $G_{\mathrm{g}}{ }^{\prime}$ and to $\Phi$ by the semi-empirical formula for closed cell foams: ${ }^{1,43}$

$$
G=\frac{9}{8} G_{\mathrm{g}}^{\prime}(1-\Phi)^{2}
$$

For our foams, we have $\Phi=0.8$ and thus $G \cong 0.045 G_{\mathrm{g}}{ }^{\prime}$. In addition, these acoustic measurements ${ }^{43}$ show that the Poisson's ratio of our foams is equal to $\nu=0.498 \pm 0.001$. With this value of $\nu$, the linearization assumption leading to eqn (3) becomes 
$\left(R_{\mathrm{O}} / L\right)^{3} \ll 0.005$ i.e. $R_{\mathrm{O}} \ll 0.2 L$. The largest investigated bubble radius is $R_{\mathrm{O}}=750 \mu \mathrm{m}$. For the smallest lateral cell dimensions (16 mm), we have $R_{\mathrm{o}} / L<0.05$. The depth of the cell is $5.3 \mathrm{~mm}$ which implies: $R_{\mathrm{O}} / L<0.14$. Thus the linearization in eqn (3) is justified, and the effect of matrix compressibility can be neglected at first order.

Our results show that the gelled foams are stable beyond $16 \mathrm{~h}$, and remain so for a few days, if the bubble radius $R_{\mathrm{O}}$ is larger than the characteristic value $R_{\mathrm{EC}}{ }^{*} \approx 500 \mu \mathrm{m}$ for $C_{\mathrm{gel}}=5 \mathrm{wt} \%$ and $R_{\mathrm{EC}}{ }^{*} \approx 150 \mu \mathrm{m}$ for $C_{\text {gel }}=10 \mathrm{wt} \%$. We deduce that in these gelled foams the elastic stresses generated by the deformation of the bubbles are strong enough to oppose a change in bubble size due to interbubble diffusion of nitrogen. Thus we identify each one of these radii to the elasto-capillary radius that is characteristic of a foam with a surface tension $\gamma$ and a matrix elasticity $G$ evaluated at $t_{\max }=16 \mathrm{~h}$ for each gelatin concentration. The specific choice of this age is not crucial since on such long time scales, the evolution of the gel storage modulus $G_{\mathrm{g}}{ }^{\prime}$ is logarithmic ( $c f$. Fig. 2). We have $G_{\mathrm{g}}{ }^{\prime}=1000 \mathrm{~Pa}$, thus $G=45 \mathrm{~Pa}$ for $C_{\mathrm{gel}}=5 \mathrm{wt} \%$ and, $G_{\mathrm{g}}{ }^{\prime}=3700 \mathrm{~Pa}$, thus $G=166 \mathrm{~Pa}$ for $C_{\text {gel }}=10 \mathrm{wt} \%$.

The study of liquid foams $(G=0)$ shows that the radius at the limit of stability is of the form: $R^{*}=C R_{\mathrm{M}}$ with $C \approx 0.29$ (cf. Section 5.1). Consistently, the partial pressure of the soluble species in a bubble (eqn (14)) must be rewritten as

$$
P_{\alpha}(\varepsilon)=P_{\alpha}\left(R_{\mathrm{o}}\right)+\left(-\frac{\gamma f(\Phi)}{C^{2} R_{\mathrm{o}}}+4 g G+\frac{9 \eta_{\mathrm{o}} k T}{4 \pi R_{\mathrm{o}}{ }^{3}}\right) \varepsilon
$$

Indeed, for $G=0$, the stability criterion yields $R^{*}=C R_{\mathrm{M}}$, and for $G \neq 0$, the limit of stability $P_{\alpha}(\varepsilon)$ corresponds to $\eta_{\mathrm{o}}=\eta_{\mathrm{o}}{ }^{*}$ such that

$$
\eta_{\mathrm{o}}^{*}=\frac{16 \pi g G R_{\mathrm{o}}^{2}}{9 k T}\left(R_{\mathrm{EC}}{ }^{*}-R_{\mathrm{o}}\right)
$$

with the elasto-capillary radius

$$
R_{\mathrm{EC}}^{*}=\frac{\gamma f(\Phi)}{4 g C^{2} G}
$$

Thus, from eqn (22), we deduce the relation: $g=\gamma f(\Phi) /\left(4 C^{2} G R_{\mathrm{EC}}{ }^{*}\right)$. We recall that the parameters $\gamma=0.031 \mathrm{~N} \mathrm{~m}^{-1}$ and $f(\Phi)=2.0$ are identical for all studied foams. For $C_{\text {gel }}=5 \mathrm{wt} \%$, we have $G=45 \mathrm{~Pa}$ and $R_{\mathrm{EC}}{ }^{*} \approx 500 \mu \mathrm{m}$, which yields $g \approx 7.7$. For $C_{\text {gel }}=10 \mathrm{wt} \%$, we have $G=166 \mathrm{~Pa}$ and $R_{\mathrm{EC}}{ }^{*} \approx 150 \mu \mathrm{m}$, which yields $g \approx 7.0$. Both estimations of the factor $g$ are close to each other and the factor $g C^{2}$ is of the order of one as expected for an ideal foam $R_{\mathrm{EC}}{ }^{*} \approx R_{\mathrm{EC}}$. In the following, we take: $g=8$. With this value and eqn (21), we predict the surface delimiting the stable foam domain in a $3 \mathrm{D}$ phase diagram where the space parameters are $\left(R_{\mathrm{o}}, G\right.$ and $\left.\eta_{\mathrm{o}}\right)$. Then, all of our data points corresponding to unstable foams should lie below this surface, and those for stable foams should be located above it. To test this conjecture, we plot all of our data for both liquid and gelled foams in such a diagram in Fig. 13a. Fig. 13b shows a zoom of the region for small $G$ and $\eta_{\mathrm{o}}$. As can be seen, the surface $\eta_{\mathrm{o}}{ }^{*}\left(R_{\mathrm{o}}, G\right)$ delimitates the frontier between stable and unstable foams. This result demonstrates that our model based on an ideal
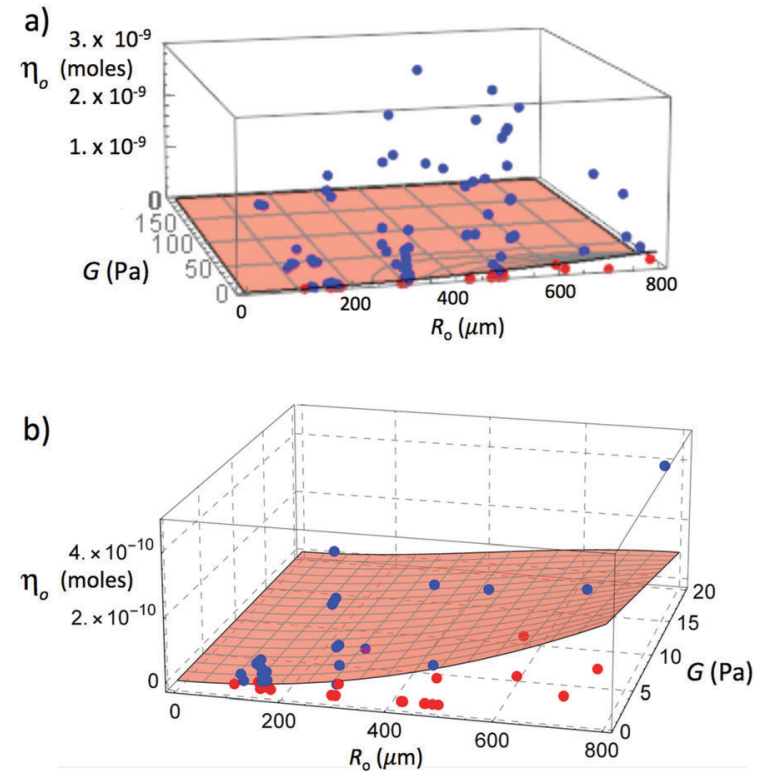

Fig. 13 (a) 3D phase diagram showing stable (blue $O$ ) or unstable (red $O$ ) gelled and liquid foams as a function of the initial bubble radius $R_{0}$, the number of moles $\eta_{\circ}$ of insoluble $\mathrm{C}_{6} \mathrm{~F}_{14}$ gas molecules per bubble, and the foam storage modulus $G$. The continuous surface represents eqn (21) with eqn (22), $C=0.29$ and the factor $g=8$ (see text). The unstable region is located below the surface. (b) Zoom of the phase diagram to show the region of small $\eta_{\circ}$ and $G$. The equation of the surface is the same as in (a). The experimental parameters are $\Phi=0.8, f(\Phi)=2.0, \gamma=0.031 \mathrm{mN} \mathrm{m}^{-1}$, and $T=295 \mathrm{~K}$.

foam structure captures the underlying mechanisms of foam stabilisation by the combined effects of coarsening inhibition by an insoluble gaseous species and elasticity of the continuous phase. Numerical simulations would be of great interest to take into account the details of the foam microstructure and to quantitatively predict the prefactor $g$.

\section{Conclusions}

By elaborating monodispersed gelled foams with controlled gas mixtures, we are able to investigate the combined effects of a gas species trapped inside the bubbles and of gelation on foam stabilization. We identify the underlying physical mechanisms that lead to coarsening inihibition: the osmotic effect due to the partial pressure of the insoluble species tends to stabilize foams with small bubbles as it damps bubble size fluctuations, in contrast to the capillary pressure that tends to amplify them. Restoring elastic stresses generated by bubble deflation or inflation can counteract the capillary pressure differences between bubbles and stabilize the foam. We predict the stability diagram of an ideal monodisperse ordered foam as a function of the bubble radius $R_{\mathrm{o}}$, the number of insoluble molecules per bubble $\eta_{\mathrm{o}}$ (or the partial pressure of the insoluble species $P_{\eta}$ ), and the storage shear modulus of the gelled foam $G$.

Liquid foams solely stabilized by the presence of the insoluble species are studied as a reference case. This allows us to identify the domain of stable foams in the parameter space 
$\left(R_{\mathrm{o}}, \sqrt{\eta_{\mathrm{o}}}\right)$ or $\left(R_{\mathrm{o}}, P_{\eta}\right)$. The comparison between our data and this prediction shows that the bubble radius at the stability frontier does scale as $\sqrt{\eta_{\mathrm{o}}}$ or $1 / P_{\eta}$ as expected based on scaling arguments. However, real foams appear to be less stable than ideal ones: for a given $R_{\mathrm{o}}$, more insoluble gas is required to stabilize our real foam than an ideal one. It is likely that this difference is due to the disorder in polydispersity and topology. Further theoretical work is needed to modelize the impact of disorder on foam coarsening inhibition.

Gelled foams are prepared from gelatin solutions at high temperature, and then quenched at room temperature where physical cross-links progressively form an elastic network. The storage modulus of the gelifying solution is measured independently as the solution undergoes the same thermal history as that in the quenched foam. We map our data on a stability diagram where the control parameters are $\left(R_{\mathrm{o}}, \eta_{\mathrm{o}}\right.$ and $\left.G\right)$. We show that coarsening can be inhibited for the bubble radius $R_{\mathrm{O}}$ larger than a critical elasto-capillary radius (eqn (22)) set by the ratio of surface tension to foam storage modulus. Despite the presence of structural defects, the observed elasto-capillary radius is of the same order of magnitude as that of an ideal ordered structure. Foams with smaller bubbles can be stable in the presence of an insoluble species if the number of trapped molecules in a bubble $\eta_{\mathrm{o}}$ is large enough as given by eqn (21). The qualitative agreement between our data and the diagram predicted for an ideal foam demonstrates that the underlying physical ingredients are well captured by our scaling model. Numerical simulations and further theoretical modelization would be useful to take into account foam polydispersity or more realistic details of the foam microstructure in the coarsening process and in the elastic response accompanying changes of bubble volumes in order to capture the key structural parameters at play. Modelization and simulations of the kinetics of the bubble size evolution under the combined effects of the trapped gas species and gelation would be interesting future developments.

To conclude, this work should be useful to elaborate stable foams where coarsening is inhibited either by the presence of a trapped gaseous species insoluble in the foaming liquid or by elastic stresses in the matrix, or by both effects. This will be useful for many applications in materials science, in particular, for the elaboration of solid foams from liquid precursors.

\section{Appendix}

\section{Annex: transfer of the weakly soluble species $\alpha$ between the gas and the liquid phases}

The foam is produced using water that is initially saturated with air at atmospheric ambient pressure $P_{\mathrm{o}}$. The partial pressure of $\alpha$ (nitrogen in our case) in the atmosphere is denoted as $P_{\mathrm{o}, \alpha}$. Thus the initial concentration of the species $\alpha$ in the liquid is $C_{\infty}=H_{\alpha} P_{\mathrm{o}, \alpha}$, where $H_{\alpha}$ is Henry's law constant (cf. Section 2.3). In the foam, the chemical equilibrium requires that the chemical potentials of the species $\alpha$ are equal in the gas and liquid phases. This condition is fulfilled when the concentration of $\alpha$ in the liquid around a bubble of radius $R_{\mathrm{O}}$ is given by

$$
C_{\mathrm{o}}=C_{\infty} \frac{P_{\alpha}\left(R_{\mathrm{o}}\right)}{P_{\mathrm{o}, \alpha}}
$$

where $P_{\alpha}\left(R_{\mathrm{O}}\right)$ is the partial pressure of the species $\alpha$ in a bubble of radius $R_{\mathrm{O}}$ as given by eqn (10).

When $C_{\mathrm{o}} \neq C_{\infty}$, there is a transfer of the species $\alpha$ between the gas phase and the liquid in order to establish the equilibrium concentration of $\alpha$ in the liquid. In a volume $V$ of foam with gas volume fraction $\Phi$, the bubbles must lose or gain a number of molecules $\Delta n_{\alpha, g}$ :

$$
\Delta n_{\alpha, \mathrm{g}}=\left|C_{\mathrm{o}}-C_{\infty}\right|(1-\Phi) V
$$

The number $n_{\alpha, g}$ of molecules available in the gas phase in the same foam volume $V$ is

$$
n_{\alpha, \mathrm{g}}=\frac{\Phi V P_{\alpha}\left(R_{\mathrm{o}}\right)}{k T}
$$

where $k$ is the Boltzmann constant and $T$ is the temperature. Combining eqn (23)-(25), we deduce the relative variation of the number of $\alpha$ molecules in a bubble:

$$
\frac{\Delta n_{\alpha, \mathrm{g}}}{n_{\alpha, \mathrm{g}}}=k T H_{\alpha} \frac{1-\Phi}{\Phi}\left|1-\frac{P_{\mathrm{o}, \alpha}}{P_{\alpha}\left(R_{\mathrm{o}}\right)}\right|
$$

Thus, the amount of transferred gas remains small since Henry's solubility constant $H_{\alpha}$ of the species $\alpha$ is small.

In our experiments, we have the parameters: $P_{\mathrm{o}, \alpha}=0.8$ bar, $\Phi=0.8, H_{\alpha}=6.4 \times 10^{-6} \mathrm{~mol} \mathrm{~m}^{-3} \mathrm{~Pa}^{-1}$ and $T=22^{\circ} \mathrm{C}$. In the range of investigated $P_{\eta}$ and $R_{\mathrm{o}}, P_{\alpha}\left(R_{\mathrm{o}}\right)$ varies between extremal values that are close to $P_{\mathrm{o}, \alpha}$ and $P_{\mathrm{o}}$. According to eqn (24), we have in this range: $\Delta n_{\alpha, g} / n_{\alpha, g}<0.001$. Thus, the amount of transferred gas is indeed very small and we neglect the variation of the bubble radius it induces.

\section{Acknowledgements}

We thank Tristan Baumberger and Christiane Caroli for their fruitful suggestions. H. Bey gratefully acknowledges financial support from the Ministry of Higher Education and Scientific Research in Tunisia (MHESRT) for financial support. We gratefully acknowledge financial support from the Centre National d'Etudes Spatiales (convention de recherches CNES/CNRS 160483) and the European Space Agency ("Soft Matter Dynamics" MAP grant AO-09-943).

\section{References}

1 L. J. Gibson and M. F. Ashby, Cellular solids, Cambridge University Press, 2nd edn, 1997.

2 M. Scheffler and P. Colombo, Cellular Ceramics: Structure, Manufacturing, Properties and Applications, Wiley-VCH, 2005.

3 J. Banhart, Adv. Eng. Mater., 2006, 8, 781-794.

4 G. Lan, B. Lu, T. Wang, L. Wang, J. Chen, K. Yu, J. Liu, F. Dai and D. Wu, Colloids Surf., B, 2015, 136, 1026-1034. 
5 K.-Y. Chung, N. C. Mishra, C.-C. Wang, F.-H. Lin and K.-H. Lin, Biomicrofluidics, 2009, 3, 022403.

6 A. Barbetta, G. Rizzitelli, R. Bedini, R. Pecci and M. Dentini, Soft Matter, 2010, 6, 1785-1792.

7 M. Costantini, C. Colosi, J. Jaroszewicz, A. Tosato, W. Swieszkowski, M. Dentini, P. Garstecki and A. Barbetta, ACS Appl. Mater. Interfaces, 2015, 7, 23660-23671.

8 R. Deleurence, T. Saison, F. Lequeux and C. Monteux, Soft Matter, 2015, 11, 7032-7037.

9 I. Cantat, S. Cohen-Addad, F. Elias, F. Graner, R. Höhler, O. Pitois, F. Rouyer and A. Saint-Jalmes, Foams: Structure and Dynamics, Oxford University Press, Oxford, 2013.

10 U. T. Gonzenbach, A. R. Studart, E. Tervoort and L. J. Gauckler, Angew. Chem., Int. Ed., 2006, 45, 3526-3530.

11 E. Rio, W. Drenckhan, A. Salonen and D. Langevin, Adv. Colloid Interface Sci., 2014, 205, 74-86.

12 I. Lesov, S. Tcholakova and N. Denkov, J. Colloid Interface Sci., 2014, 426, 9-21.

13 R. M. Guillermic, A. Salonen, J. Emile and A. Saint-Jalmes, Soft Matter, 2009, 5, 4975-4982.

14 B. Haffner, Y. Khidas and O. Pitois, J. Colloid Interface Sci., 2015, 458, 200-208.

15 T. Li, F. Nudelman, J. W. Tavacoli, H. Vass, D. J. Adams, A. Lips and P. S. Clegg, Adv. Mater. Interfaces, 2016, 3, 1500601.

16 S. Cohen-Addad, R. Höhler and O. Pitois, Annu. Rev. Fluid Mech., 2013, 45, 241-267.

17 A. Testouri, C. Honorez, A. Barillee, D. Langevin and W. Drenckhan, Macromolecules, 2010, 43, 6166-6173.

18 A. Testouri, L. R. Arriaga, C. Honorez, M. Ranft, J. Rodrigues, A. van der Net, A. Lecchi, A. Salonen, E. Rio, R. M. Guillermic, D. Langevin and W. Drenckhan, Colloids Surf., A, 2012, 413, 17-24.

19 W. Kloek, T. van Vliet and M. Meinders, J. Colloid Interface Sci., 2001, 237, 158-166.

20 R. Höhler, Y. Yip Cheung Sang, E. Lorenceau and S. Cohen-Addad, Langmuir, 2008, 24, 418-425.

21 M. Le Merrer, S. Cohen-Addad and R. Hohler, Phys. Rev. Lett., 2012, 108, 188301.

22 S. Tcholakova, Z. Mitrinova, K. Golemanov, N. D. Denkov, M. Vethamuthu and K. P. Ananthapadmanabhan, Langmuir, 2011, 27, 14807-14819.
23 A. Salonen, C. Gay, A. Maestro, W. Drenckhan and E. Rio, EPL, 2016, 116, 46005.

24 D. Weaire and V. Pageron, Philos. Mag. Lett., 1990, 62, 417-421.

25 A. J. Webster and M. E. Cates, Langmuir, 1998, 14, 2068-2079.

26 A. J. Webster and M. E. Cates, Langmuir, 2001, 17, 595-608.

27 F. G. Gandolfo and H. L. Rosano, J. Colloid Interface Sci., 1997, 194, 31-36.

28 P. Stevenson, Foam Engineering: Fundamentals and Applications, J. Wiley, 2012.

29 V. Sovilj, M. Jadranka and L. Petrovic, Hydrocolloids, 2013, 32, 20-27.

30 R. Sander, Atmos. Chem. Phys., 2015, 15, 4399-4981.

31 Y. Yip Cheung Sang, PhD thesis, University Paris Diderot, 2009.

32 Y. Yip Cheung Sang, E. Lorenceau, S. Wahl, M. Stoffel, D. E. Angelescu and R. Höhler, RSC Adv., 2013, 3, 2330-2335.

33 H. Souguir, O. Ronsin, V. Larreta-Garde, T. Narita, C. Caroli and T. Baumberger, Soft Matter, 2012, 8, 3363-3373.

34 K. te Nijenhuis, Thermoreversible Networks, Springer, Berlin, 1997, vol. 130.

35 O. Ronsin, C. Caroli and T. Baumberger, Phys. Rev. Lett., 2009, 103, 138302.

36 A. K. Mal and S. J. Singh, Deformation of Elastic Solids, Prentice Hall, London, 1991.

37 M. Fyrillas, W. Kloek, T. van Vliet and J. Mellema, Langmuir, 2000, 16, 1014-1019.

38 S. Tcholakova, N. D. Denkov, K. Golemanov, K. P. Ananthapadmanabhan and A. Lips, Phys. Rev. E: Stat., Nonlinear, Soft Matter Phys., 2008, 78, 011405.

39 B. Roman and J. Bico, J. Phys.: Condens. Matter, 2010, 22, 493101.

40 A. Kabalnov, D. Klein, T. Pelura, E. Schutt and J. Weers, Ultrasound in Medicine and Biology, 1998, 24, 739-749.

41 S. Shim, J. D. Wan, S. Hilgenfeldt, P. D. Panchal and H. A. Stone, Lab Chip, 2014, 14, 2428-2436.

42 I. Jorjadze, L.-L. Pontani and J. Brujic, Phys. Rev. Lett., 2013, 110, 048302.

43 F. Wintzenrieth, PhD thesis, University Pierre et Marie Curie, 2015. 